\title{
Motion and properties of nuclear radio components in Seyfert galaxies seen with VLBI
}

\author{
E. Middelberg ${ }^{1}$, A. L. Roy ${ }^{1,7}$, N. M. Nagar ${ }^{2,9}$, T. P. Krichbaum ${ }^{1}$, R. P. Norris ${ }^{3}$, A. S. Wilson ${ }^{4}$, H. Falcke ${ }^{1,8}$, \\ E. J. M. Colbert ${ }^{5}$, A. Witzel ${ }^{1}$, and K. J. Fricke ${ }^{6}$ \\ 1 Max-Planck-Institut für Radioastronomie, Auf dem Hügel 69, 53121 Bonn, Germany \\ e-mail: emiddelb; aroy; tkrichbaum; awitzel@mpifr-bonn.mpg.de \\ 2 Kapteyn Institute, University of Groningen, Landleven 12, 9747 AD Groningen, The Netherlands \\ e-mail: nagar@astro.rug.nl \\ 3 Australia Telescope National Facility, PO Box 76, Epping, NSW, Australia \\ e-mail: rnorris@atnf.csiro.au \\ 4 Department of Astronomy, University of Maryland, College Park, MD 20742, USA \\ e-mail: wilson@astro.umd.edu \\ 5 John Hopkins University, Department of Physics and Astronomy, 3400 North Charles Street, Baltimore, MD 21218, USA \\ e-mail: colbert@pha.jhu.edu \\ ${ }^{6}$ Universitäts-Sternwarte, Geismarlandstrasse 11, 37083 Göttingen, Germany \\ e-mail: kfricke@uni-sw.gwdg.de \\ 7 Geodätisches Institut der Universität Bonn, Nussallee 17, 53115 Bonn, Germany \\ 8 ASTRON, PO Box 2, 7990 AA Dwingeloo, The Netherlands \\ e-mail: falcke@astron.nl \\ 9 INAF, Arcetri Observatory, Largo E. Fermi 5, Florence 50125, Italy
}

Received 28 November 2002 / Accepted 14 January 2004

\begin{abstract}
We report EVN, MERLIN and VLBA observations at $18 \mathrm{~cm}, 6 \mathrm{~cm}$ and $3.6 \mathrm{~cm}$ of the Seyfert galaxies NGC 7674, NGC 5506, NGC 2110 and Mrk 1210 to study their structure and proper motions on pc scales and to add some constraints on the many possible causes of the radio-quietness of Seyferts. The component configurations in NGC 7674 and NGC 2110 are simple, linear structures, whereas the configurations in NGC 5506 and Mrk 1210 have multiple components with no clear axis of symmetry. We suggest that NGC 7674 is a low-luminosity compact symmetric object. Comparing the images at different epochs, we find a proper motion in NGC 7674 of $(0.92 \pm 0.07) c$ between the two central components separated by $282 \mathrm{pc}$ and, in NGC 5506, we find a $3 \sigma$ upper limit of $0.50 \mathrm{c}$ for the components separated by $3.8 \mathrm{pc}$. Our results confirm and extend earlier work showing that the outward motion of radio components in Seyfert galaxies is non-relativistic on pc scales. We briefly discuss whether this non-relativistic motion is intrinsic to the jet-formation process or results from deceleration of an initially relativistic jet by interaction with the pc or sub-pc scale interstellar medium. We combined our sample with a list compiled from the literature of VLBI observations made of Seyfert galaxies, and found that most Seyfert nuclei have at least one flat-spectrum component on the VLBI scale, which was not seen in the spectral indices measured at arcsec resolution. We found also that the bimodal alignment of $\mathrm{pc}$ and kpc radio structures displayed by radio galaxies and quasars is not displayed by this sample of Seyferts, which shows a uniform distribution of misalignment between $0^{\circ}$ and $90^{\circ}$. The frequent misalignment could result from jet precession or from deflection of the jet by interaction with gas in the interstellar medium.
\end{abstract}

Key words. galaxies: seyfert - galaxies: active - galaxies: jets

\section{Introduction}

Active galaxies are conventionally divided into radio-quiet and radio-loud categories. Kellermann et al. (1989) found that quasars separated into these two classes when the ratio of their radio to optical continuum was considered, and a similar result has been found by most (e.g. Peacock et al. 1986; Miller et al. 1990; Ivezić et al. 2002) but not all (White et al. 2000)

Send offprint requests to: E. Middelberg, e-mail: emiddelb@mpifr-bonn.mpg.de workers since. Members of the radio-loud category include BL Lac objects, radio galaxies and radio-loud quasars. Radioloud objects have large-scale radio lobes and compact luminous cores which often exhibit apparent superluminal motion. Radio-quiet objects include Seyfert galaxies and radio-quiet quasars; most of their radio emission is confined to the sub-kpc scale.

Recent Very Long Baseline Interferometry (VLBI) has shown that many Seyferts also contain compact nuclear radio components with brightness temperatures $\gg 10^{7} \mathrm{~K}$ despite 
having radio luminosities over a factor of $10^{8}$ less than the most powerful active galaxies (e.g. Ulvestad et al. 1998; Ulvestad et al. 1999a; Ulvestad et al. 1999b; Roy et al. 2000; Mundell et al. 2000), and that they harbour compact components moving at non-relativistic speeds. In all cases, the central masses are inferred to be black holes of millions to billions of solar masses, so the central potentials are relativistically deep. Since most accretion energy is released close to the hole, jets might be expected to be relativistic in all cases. Why then are the radio sources in Seyferts small, weak and slow compared to those in radio-loud objects?

Various answers to this question have been proposed, involving either "intrinsic" differences in the central engine or "extrinsic" differences in the surrounding medium. Intrinsic differences that have been suggested include (i) systematically lower black hole masses in Seyferts (Laor 2000), (ii) lower black hole spins (Wilson \& Colbert 1995), (iii) a "magnetic switch" that was identified by Meier et al. (1997) during numerical modelling of jets, (iv) the production in Seyferts of buoyant plasmons that bubble up through the density gradient of the narrow line region (NLR) instead of a collimated relativistic jet (Pedlar et al. 1985; Whittle et al. 1986; Taylor et al. 1989), (v) a large thermal plasma fraction in the jet in Seyferts (Bicknell et al. 1998), or (vi) radiative inefficiency (Falcke \& Biermann 1995). Extrinsic differences generally invoke the rapid deceleration of initially relativistic jets by collisions in a dense surrounding BLR or ISM (e.g. Norman \& Miley 1984). With so many possible causes, and so few observational constraints, the question remains open why Seyfert galaxies have such low radio luminosities.

One approach to investigate whether the radio quietness of Seyferts is due to intrinsic or extrinsic causes is to measure speeds of radio components as close to the nucleus as possible, hopefully before interactions with the ISM affect them much. How close that needs to be is unclear, but signs of jet-NLR interaction have been seen on scales of tens of pc (Whittle et al. 1986; Wilson \& Tsvetanov 1994; Bower et al. 1995; Capetti et al. 1997; Falcke et al. 1998), so sub-pc scales are desirable. Proper motions of Seyfert galaxy radio components have now been measured with VLBI in a few cases (e.g. Mrk 231 and Mrk 348 by Ulvestad et al. 1999b, NGC 1068 by Roy et al. 2000, and NGC 4151 by Ulvestad et al. 1998; Ulvestad et al. 2002), and the motions were found to be $\leq 0.25 c$. In III Zw 2 , $v<0.04 c$ was seen between two barely-resolved components over eight months, followed by a sudden displacement at $v \geq 1.25 c$ over seven months (Brunthaler et al. 2000).

The observations presented here were made for three reasons: 1) to measure proper motions of radio components on pc scales; 2) to look for extended, low surface-brightness radio emission on the scale of the NLR. In the event that slow proper motions were measured, the next question would be whether components were ejected slowly or whether they were ejected at high, perhaps relativistic speeds and then slowed by interaction with the NLR gas. Were such braking to occur, one would expect shocks in the jet and possibly jet disruption. The in-situ particle acceleration associated with shocks could lead then to extended regions of radio emission; 3) to measure spectral indices, which provide a useful diagnostic of emission mechanisms, source compactness, and possible foreground free-free absorption. Most spectral index measurements of Seyfert galaxies to date have used the VLA, ATCA or WSRT, whose relatively large beams can average together many components in the nucleus. Measurements with parsecscale resolution in Seyfert galaxies enable one to separate a possible compact, flat-spectrum component from surrounding steep-spectrum, optically-thin synchrotron emission, but such measurements require VLBI with matched beams at two frequencies and are still relatively few. In contrast, powerful radio sources have been well studied with VLBI and flat-spectrum core components are often seen at the base of a conicallyexpanding jet, thus indicating the location of the nucleus. Do Seyfert nuclei also show a single, compact, synchrotron selfabsorbed core component at the base of a collimated jet?

To meet these goals, we observed using Global VLBI, the European VLBI Network (EVN), the Multi-Element Radio Linked Interferometer Network (MERLIN) and the Very Long Baseline Array (VLBA) the Seyfert galaxies NGC 5506 and NGC 7674 at multiple epochs for proper motion measurements of nuclear radio sources, and Mrk 1210 and NGC 2110 for the first epoch. To enlarge the sample size for deriving statistics on the spectral and geometric properties of the pc scale structure of Seyferts, we made a complete literature review of VLBI observations of Seyfert galaxies. We assume $H_{0}=75 \mathrm{~km} \mathrm{~s}^{-1} \mathrm{Mpc}^{-1}$ and $q_{0}=0.5$ throughout this paper, and calculate spectral indices using $S \propto v^{\alpha}$.

\section{The sample and observations}

The galaxies presented here are part of a sample selected for global VLBI observations in 1994 to investigate the dichotomy between radio-loud and radio-quiet AGN. The sample comprised 16 Seyfert 2 galaxies from Véron-Cetty \& Véron (1991) with $6 \mathrm{~cm}$ core flux densities $>70 \mathrm{mJy}$ and $\delta>-10^{\circ}$. The literature was then carefully reviewed for updated spectral classifications, some of the objects were discarded and others were incorporated based on their new classification and flux density measurements.

Global VLBI, 1994. The sample was observed in 1994 with global VLBI at $6 \mathrm{~cm}$, using the ten VLBA stations, the phased VLA, Effelsberg, Onsala, Medicina and Noto for $36 \mathrm{~min}$ in six snapshots over $11 \mathrm{~h}$, yielding a $\sim 30 \mathrm{~min}$ integration of each object (Krichbaum et al. 1994, unpublished). The observations yielded only the detection of point sources in the cases of NGC 7674, NGC 2110 and Mrk 1210, and lacked short baselines and spectral index information. Only the NGC 5506 data are included in this paper.

EVN and MERLIN, 1999/2000. Four of those objects (NGC 5506, NGC 7674, NGC 2110 and Mrk 1210) were reobserved in 1999 and 2000 with better sensitivity and better $(u, v)$-plane coverage using EVN and MERLIN at $18 \mathrm{~cm}$ and $6 \mathrm{~cm}$.

The observations were made with eight EVN stations (Effelsberg, Cambridge $32 \mathrm{~m}$, Jodrell Bank Mk 1 at $18 \mathrm{~cm}$ and Jodrell Bank Mk 2 at $6 \mathrm{~cm}$, Medicina, Noto, Onsala $25 \mathrm{~m}$, Westerbork array, Torun) at $18 \mathrm{~cm}(1.65 \mathrm{GHz})$ on November 11, 1999, and at $6 \mathrm{~cm}(4.99 \mathrm{GHz})$ on 
Table 1. List of observed sources. Columns 2 and 3 give the coordinates used in the observations and for correlation, Col. 4 gives the source distances in Mpc. Column 5 gives the classification according to Véron-Cetty \& Véron (2001).

\begin{tabular}{|c|c|c|c|c|c|c|}
\hline Source & RA (J2000) & Dec (J2000) & $D$ & Type & Purpose & Position reference \\
\hline NGC 5506 & 141314.87926 & -031227.6514 & $24.7^{1 a)}$ & $\mathrm{S} 1 \mathrm{n}^{1 b)}$ & Science Target & Roy et al. $(1997)^{1 c)}$ \\
\hline $1404-015$ & 140445.8949 & -013021.937 & & & Phase calibrator & - \\
\hline NGC 7674 & 232756.712 & +084644.14 & $116^{2 a)}$ & $\mathrm{S} 1 \mathrm{~h}^{2 b)}$ & Science Target & Krichbaum et al. $(1994)^{2 c)}$ \\
\hline $2327+096$ & 232733.5808 & +094009.460 & & & Phase calibrator & Browne et al. (1998) \\
\hline NGC 2110 & 055211.376 & -072722.52 & $31.1^{3 a)}$ & $\mathrm{S} 1 \mathrm{i}^{3 b)}$ & Science Target & Ulvestad \& Wilson $(1983)^{3 c)}$ \\
\hline $0541-056$ & 054138.0848 & -054149.395 & & & Phase calibrator & - \\
\hline Mrk 1210 & 080405.856 & +050649.83 & $53.9^{4 a)}$ & $\mathrm{S} 1 \mathrm{~h}^{4 b)}$ & Science Target & Falcke et al. $(1998)^{4 c)}$ \\
\hline $0803+043$ & 080356.4444 & +042102.724 & & & Phase calibrator & Browne et al. (1998) \\
\hline
\end{tabular}

Remarks: 1a) Keel (1996); 1b) Nagar et al. (2002b); 1c) Coordinates from Roy et al. (1997) observations (unpublished), which based on VLA observations by Ulvestad \& Wilson (1984); 2a) Nishiura et al. (2000); 2b) Véron-Cetty \& Véron (2001); 2c) VLA 6 cm (unpublished), 232524.410 +08 3012.60 (B1950) precessed to J2000 by NRAO's scheduling software SCHED, Keel (1996); 3a) Nelson \& Whittle (1995); 3b) Véron-Cetty \& Véron (1998); 3c) 054946.376 -07 2801.99 (B1950) precessed to J2000 by SCHED; 4a) de Vaucouleurs et al. (1991); 4b) Véron-Cetty \& Véron (1998); 4c) Based on $6 \mathrm{~cm}$ VLA observations from Falcke et al. (1998).

February 28, 2000. The $18 \mathrm{~cm}$ EVN observations were recorded in MkIII mode with a bandwidth of $56 \mathrm{MHz}, 1$ bit sampling and dual circular polarization, and were correlated in Bonn on the MkIII correlator. The $6 \mathrm{~cm}$ EVN observations were recorded in the new MkIV mode with a bandwidth of $32 \mathrm{MHz}, 2$ bit sampling and dual circular polarization, and were correlated at the Joint Institute for VLBI in Europe (JIVE). The MERLIN observations were correlated in real time with a bandwidth of $32 \mathrm{MHz}, 2$ bit sampling and dual circular polarization. As all sources are weak, phasereferencing was used for initial phase calibration. Details of the calibrators are given in Table 1 . All calibrators lie within $6^{\circ}$ of the targets. Phase calibrator scans were obtained every $5 \mathrm{~min}$ to $10 \mathrm{~min}$ at $18 \mathrm{~cm}$ and every $4 \mathrm{~min}$ at $6 \mathrm{~cm}$.

Additional data. Further observations presented in this paper were done in the framework of other projects:

- NGC 5506 was observed during two further VLBI experiments, designed for proper motion measurement.

(i) February 5, 1997: NGC 5506 was observed with the ten VLBA stations at $18 \mathrm{~cm}, 6 \mathrm{~cm}$ and $3.6 \mathrm{~cm}$ with onsource times of $91 \mathrm{~min}, 64 \mathrm{~min}$ and $43 \mathrm{~min}$, a bandwidth of $32 \mathrm{MHz}, 2$ bit sampling and right circular polarization.

(ii) May 31, 2000: NGC 5506 was observed with the ten VLBA stations at $18 \mathrm{~cm}$ and $6 \mathrm{~cm}$ with on-source times of 204 min at each wavelength, a bandwidth of $32 \mathrm{MHz}$, 2 bit sampling and left circular polarization.

The 1994 and 2000 VLBA observations and the EVN observations were reduced in AIPS using the same procedure for each (see below), including the same weighting, taper and restoring beam size, and the 1997 VLBA observations were reduced using the same methods except for slightly different tapering. All $18 \mathrm{~cm}$ and $6 \mathrm{~cm}$ images are shown in Fig. 1.

- NGC 7674 was observed at $18 \mathrm{~cm}$ on April 13, 1985 by Unger et al. (1988) with four EVN stations using a bandwidth of $28 \mathrm{MHz}$ and dual circular polarization.
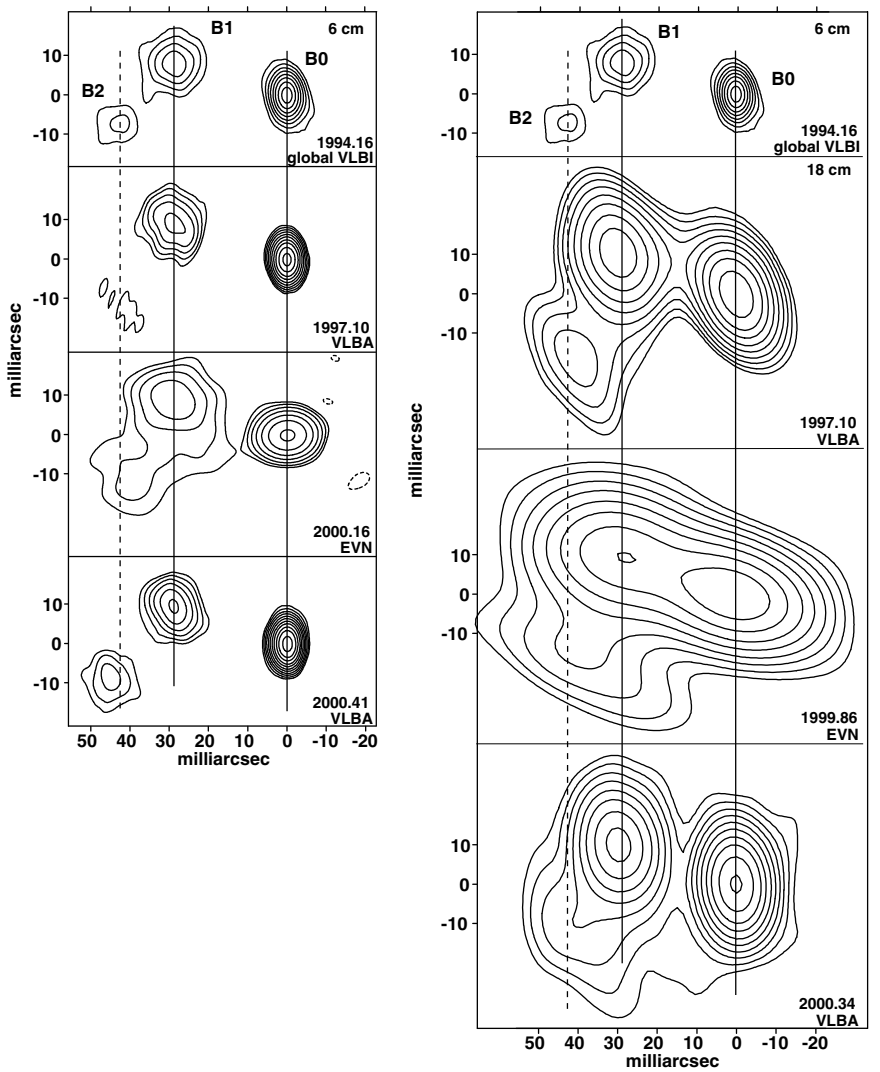

Fig. 1. VLBI $6 \mathrm{~cm}$ images at four epochs (left panels), from which we placed an upper limit on the proper motion of the B1 component, relative to B0, in NGC 5506. The proper motion of the B2 component is unreliable due to limited image fidelity. The $6 \mathrm{~cm} 1994.16$ epoch is repeated as the top right panel. The lower three right panels show the $18 \mathrm{~cm}$ images. All images are naturally weighted. The VLBA observations were restored with common beams of 7.4 mas $\times 4.3$ mas $(6 \mathrm{~cm})$ and $18.5 \mathrm{mas} \times 11.3$ mas $(18 \mathrm{~cm})$.

We re-reduced those data from the archive in Bonn following the same procedures as for the $1999 \mathrm{EVN} 18 \mathrm{~cm}$ data, yielding images with $\mathrm{rms}=0.46 \mathrm{mJy} \mathrm{beam}^{-1}$ and a beam 
size of 39.3 mas $\times 24.2$ mas. Amplitude calibration data were not archived, so we used $T_{\text {sys }}$ and gain measurements from the immediately preceding experiment (Porcas, priv. comm.).

- Mrk 1210 was observed on August 6, 1998 with the VLBA at $18 \mathrm{~cm}$ and $6 \mathrm{~cm}$ with on-source times of $160 \mathrm{~min}$ and $140 \mathrm{~min}$, a bandwidth of $32 \mathrm{MHz}, 2$ bit sampling and dual circular polarization. Phase-referencing was made to the same calibrator as for the EVN and MERLIN observations. The observations yielded thermal noise limited images with resolutions of 18.6 mas $\times 14.2$ mas and 3.9 mas $\times$ 1.8 mas at $18 \mathrm{~cm}$ and $6 \mathrm{~cm}$.

Data reduction was done using the Astronomical Image Processing System (AIPS). The EVN and VLBA amplitudes were calibrated using $T_{\text {sys }}$ measurements and the MERLIN amplitudes were calibrated using the flux density calibrator DA 193 assuming flux densities of $2.12 \mathrm{Jy}$ at $18 \mathrm{~cm}$ and $5.15 \mathrm{Jy}$ at $6 \mathrm{~cm}$. Residual delays and phases were determined by fringe fitting on the phase reference calibrators and interpolating the solutions onto the targets. If the target was visible in the resulting phase-referenced image, one to three iterations of phase self-calibration and deconvolution using CLEAN were performed until a thermal noise limited image was achieved. Absolute position information is then preserved as the self-calibration uses phase-referenced, and therefore position-referenced, model components. If the target was not visible after phase referencing, the data were self-calibrated using a point source as the initial model. In this case, absolute position information was lost (see Table 2).

Absolute source positions were measured from the phasereferenced, self-calibrated images, where available; otherwise, relative source positions were measured from the selfcalibrated images. Positions were always measured by fitting a two-dimensional parabola to the source and recording the position of the peak. This was found superior to fitting Gaussians because the images of the sources were not always well described by a Gaussian. Phase-referenced positions are based on the calibrator positions in Table 1. Absolute position uncertainties are typically $10^{-7}$ times the source-calibrator separation (Lestrade 1991). Our random position uncertainties should be a small fraction of the beamwidth, and proportional to beamwidth/SNR, where SNR is the signal to noise ratio of the source component.

Flux densities were measured by integrating over the source region in the image plane. The estimated $1 \sigma$ flux density errors comprise a 5\% (MERLIN) or 10\% (other arrays) flux density scale calibration uncertainty, $\approx 0.2 \mathrm{mJy}$ additive thermal noise, the exact value depending on the source size and the particular image, and 5\% to $10 \%$ uncertainty in the integration over the source region, depending on the size and flux density. The combined estimated errors of the integrated flux densities in Table 2 represent image rms noise plus $11 \%$ $\left(S_{v}<20 \mathrm{mJy}\right)$ and $7 \%\left(S_{v}>20 \mathrm{mJy}\right)$ for MERLIN observations and $15 \%\left(S_{v}<20 \mathrm{mJy}\right)$ and $11 \%\left(S_{v}>20 \mathrm{mJy}\right)$ for other VLBI observations. The peak flux density errors are image rms noise plus 5\% for MERLIN observations and $10 \%$ for other VLBI observations.
Spectral indices were measured by tapering the $6 \mathrm{~cm}$ data and restoring with the $18 \mathrm{~cm}$ beam size. The uncertainty on the spectral indices, based on the uncertainties on the flux density measurements is $0.09(1 \sigma)$ for a $20 \mathrm{mJy}$ component. Despite the taper, some beam mismatch remained because the $18 \mathrm{~cm}$ and tapered $6 \mathrm{~cm}$ arrays were not perfectly scaled arrays; the resulting magnitude of the effect is difficult to quantify because it depends on the source structure. The largest effect probably comes from the short baselines at $18 \mathrm{~cm}$, since these were not removed to match the $6 \mathrm{~cm}$ short-baseline length. This would affect sources that have emission that is extended on scales larger than the largest angular scale to which the $6 \mathrm{~cm}$ observations are sensitive (11 mas for the EVN), but still small enough to be detected at $18 \mathrm{~cm}(<35$ mas for the EVN). The insensitivity of the $6 \mathrm{~cm}$ observations to emission on this range of scales makes the spectral index appear more negative than it actually is. We see emission extended on this scale in the case of NGC 5506 (components B0 and B1) and NGC 7674 (Momjian et al. 2003), and we attempt in these cases to estimate a correction. For the other sources our EVN $18 \mathrm{~cm}$ images and MERLIN images do not show structure extended on this scale and so we believe that no bias in the spectral indices should be present.

\section{Results and discussion}

\subsection{NGC 5506}

NGC 5506 is an edge-on, dusty irregular or early-type spiral. It has formerly been classified as a type 2 Seyfert based on the nuclear optical spectrum (Wilson et al. 1976), but recent nearIR spectra suggest that it is a narrow-line Seyfert 1 (Nagar et al. 2002b).

Colbert et al. (1996) detected large-scale radio emission extending up to $3.6 \mathrm{kpc}$ away from the disc in a north-south direction (i.e. perpendicular to the disc), but could not establish whether this is starburst or AGN driven. At higher resolution $\left(\sim 0.7^{\prime \prime}\right)$, a MERLIN $18 \mathrm{~cm}$ image by Unger et al. (1986) shows that NGC 5506 has a compact core with a diffuse halo of a few arcseconds diameter. The compact core is slightly extended and aligns with the major axis of the host galaxy and with the position angle of the optical continuum polarization (Martin et al. 1983). This is contrary to the situation in most Seyfert $2 \mathrm{~s}$, in which the polarization tends to be perpendicular to the jet axis (Antonucci \& Miller 1985) and strengthens the Sy 1 classification.

A water maser was discovered in NGC 5506 by Braatz et al. (1994) during a survey of Seyfert galaxies. Unfortunately, its weakness, $60 \mathrm{mJy}$ in the broad component, has prevented detailed VLBI imaging.

\subsubsection{Results}

Both the MERLIN $18 \mathrm{~cm}$ and $6 \mathrm{~cm}$ images (not shown) display a single unresolved point source with a total flux density of $113 \mathrm{mJy}$ and $86 \mathrm{mJy}$, respectively. The single-dish $6 \mathrm{~cm}$ flux density in a $2.4^{\prime}$ beam measured simultaneously at Effelsberg was $193 \mathrm{mJy}$, so there is significant emission on scales larger 
Table 2. Results from the observations. "Tapered" $6 \mathrm{~cm}$ flux densities were measured using images tapered and restored with the $18 \mathrm{~cm}$ beam size. The MERLIN $18 \mathrm{~cm}$ and $6 \mathrm{~cm}$ positions differ by 35 mas because the coordinates used at the correlator were changed in an unknown way and the resulting offset could not be removed accurately. We have precessed the MERLIN B1950 coordinates to J2000 using NRAO's scheduling software SCHED. Positions are absolute for observations that were phase-referenced and the phase-referencing worked; such positions are given in h, m, s (for RA) and ${ }^{\circ},{ }^{\prime},{ }^{\prime}$ (for Dec). Otherwise the positions are given in mas relative to the strongest component. The flux density error estimates represent $1 \sigma$.

\begin{tabular}{|c|c|c|c|c|c|c|}
\hline & \multirow{2}{*}{$\begin{array}{l}\text { Int. flux density } \\
(\mathrm{mJy})\end{array}$} & \multirow{2}{*}{$\begin{array}{l}\text { Peak flux density } \\
\text { (mJy/beam) }\end{array}$} & Peak position (J2000) & \multirow{2}{*}{$\begin{array}{c}\mathrm{rms} \\
(\mathrm{mJy} / \mathrm{beam})\end{array}$} & \multirow{2}{*}{$\begin{array}{l}\text { Beam size } \\
\text { (mas) }\end{array}$} & \multirow{2}{*}{$\begin{array}{c}\alpha_{6}^{18} \\
\left(S \propto v^{\alpha}\right)\end{array}$} \\
\hline & & & $\mathrm{RA} \quad \mathrm{Dec}$ & & & \\
\hline & \multicolumn{6}{|c|}{ NGC 5506} \\
\hline \multicolumn{7}{|c|}{ global VLBI, 6 cm, 28 Feb. 1994} \\
\hline B0 & $45.7 \pm 5.9$ & $33.9 \pm 4.3$ & $\begin{array}{lll}0.0 & 0.0\end{array}$ & 0.9 & $12.6 \times 6.0$ & \\
\hline $\mathrm{B} 1$ & $30.0 \pm 4.2$ & $12.2 \pm 2.1$ & $28.0 \mathrm{mas} 7.3 \mathrm{mas}$ & & & \\
\hline B2 & $10.6 \pm 2.5$ & $4.9 \pm 1.4$ & $41.6 \mathrm{mas}-8.3 \mathrm{mas}$ & & & \\
\hline \multicolumn{7}{|c|}{ VLBA 1997, $18 \mathrm{~cm}$, Feb. 5, 1997} \\
\hline B0 & $27.9 \pm 3.1$ & $24.0 \pm 2.7$ & 0.00 .0 & 0.26 & $18.4 \times 11.4$ & \\
\hline B1 & $24.4 \pm 2.9$ & $15.9 \pm 1.9$ & $29.7 \mathrm{mas} 9.7 \mathrm{mas}$ & & & \\
\hline B2 & $6.8 \pm 1.3$ & $4.0 \pm 0.7$ & $37.1 \mathrm{mas}-10.5 \mathrm{mas}$ & & & \\
\hline \multicolumn{7}{|c|}{ VLBA 1997, 6 cm, Feb. 5, 1997} \\
\hline B0 & $41.6 \pm 4.9$ & $29.6 \pm 3.3$ & $\begin{array}{lll}0.0 & 0.0\end{array}$ & 0.30 & $5.7 \times 3.7$ & \\
\hline B1 & $24.4 \pm 3.0$ & $4.3 \pm 0.7$ & 28.9 mas $9.1 \mathrm{mas}$ & & & \\
\hline \multicolumn{7}{|c|}{ VLBA 1997, 3.6 cm, Feb. 5, 1997} \\
\hline B0 & $27.0 \pm 3.4$ & $21.5 \pm 2.6$ & $\begin{array}{lll}0.0 & 0.0\end{array}$ & 0.46 & $6.5 \times 3.0$ & \\
\hline \multicolumn{7}{|c|}{ EVN, 18 cm, Nov. 11, 1999} \\
\hline B0 & $32.9 \pm 4.1$ & $31.0 \pm 3.5$ & 0.00 .0 & 0.43 & $26.2 \times 14.1$ & 0.06 \\
\hline B1 & $25.6 \pm 3.3$ & $22.1 \pm 2.6$ & 26.2 mas 9.3 mas & & & -0.73 \\
\hline B2 & $6.1 \pm 1.4$ & $5.4 \pm 1.0$ & 37.9 mas -15.2 mas & & & -0.30 \\
\hline \multicolumn{7}{|c|}{ MERLIN, $18 \mathrm{~cm}$, Nov. 11, 1999} \\
\hline & $113.2 \pm 10.3$ & $86.6 \pm 6.7$ & 0.00 .0 & 2.41 & $259.1 \times 150.9$ & -0.33 \\
\hline \multicolumn{7}{|c|}{ EVN, 6 cm, Feb. 28, 2000} \\
\hline B0 & $35.9 \pm 4.1$ & $36.1 \pm 4.2$ & $141314.87720-031227.6460$ & 0.20 & $8.8 \times 6.8$ & \\
\hline (tapered) & $32.0 \pm 3.7$ & & & & & \\
\hline $\mathrm{B} 1$ & $11.0 \pm 1.4$ & $6.8 \pm 1.2$ & $141314.87916-031227.6365$ & & & \\
\hline (tapered) & $10.8 \pm 1.4$ & & & & & \\
\hline $\mathrm{B} 2$ & $1.9 \pm 0.5$ & $1.3 \pm 0.4$ & $141314.87982-031227.6583$ & & & \\
\hline (tapered) & $4.4 \pm 0.7$ & & & & & \\
\hline \multicolumn{7}{|c|}{ MERLIN, 6 cm, Feb. 28, 2000} \\
\hline $\mathrm{B} 0+\mathrm{B} 1+\mathrm{B} 2$ & $85.8 \pm 6.4$ & $66.8 \pm 3.8$ & 0.00 .0 & 0.43 & $78.6 \times 62.4$ & \\
\hline (tapered) & $78.8 \pm 5.9$ & & & & & \\
\hline \multicolumn{7}{|c|}{ VLBA $2000,18 \mathrm{~cm}$, May 31, 2000} \\
\hline B0 & $49.0 \pm 5.8$ & $46.0 \pm 5.1$ & 0.00 .0 & 0.45 & $18.6 \times 11.3$ & \\
\hline B1 & $35.8 \pm 4.4$ & $24.7 \pm 2.9$ & 29.7 mas 10.1 mas & & & \\
\hline B2 & $4.6 \pm 1.1$ & & & & & \\
\hline \multicolumn{7}{|c|}{ VLBA $2000,6 \mathrm{~cm}$, May 31, 2000} \\
\hline B0 & $48.1 \pm 5.2$ & $42.2 \pm 4.6$ & 0.00 .0 & 0.42 & $7.4 \times 4.3$ & \\
\hline B1 & $15.3 \pm 2.7$ & $6.6 \pm 1.1$ & 29.1 mas 9.6 mas & & & \\
\hline B2 & $2.3 \pm 0.8$ & $2.7 \pm 0.7$ & $45.2 \mathrm{mas}-8.5 \mathrm{mas}$ & & & \\
\hline & & & NGC 7674 & & & \\
\hline$E V N, 18 \mathrm{cn}$ & & & & & & \\
\hline SE & $31.4 \pm 3.9$ & $31.4 \pm 3.9$ & 0.00 .0 & 0.46 & $39.3 \times 24.2$ & \\
\hline NW & $4.1 \pm 1.1$ & $5.1 \pm 0.97$ & $-453.1 \mathrm{mas} 230.8 \mathrm{mas}$ & & & \\
\hline EVN, $18 \mathrm{cn}$ & & & & & & \\
\hline SE & $37.6 \pm 4.5$ & $23.9 \pm 2.8$ & $232756.71054+084644.1310$ & 0.40 & $32.5 \times 9.8$ & -1.78 \\
\hline NW & $11.0 \pm 2.1$ & $5.6 \pm 1.0$ & $232756.67984+084644.3641$ & & & \\
\hline MERLIN, & & & & & & \\
\hline SE & $111.5 \pm 8.6$ & $74.9 \pm 4.6$ & $232756.71730+084644.0104$ & 0.82 & $276 \times 110$ & -1.45 \\
\hline NW & $45.6 \pm 4.0$ & $29.2 \pm 2.3$ & $232756.68608+084644.2195$ & & & -1.58 \\
\hline EVN, $6 \mathrm{~cm}$ & & & & & & \\
\hline $\mathrm{SE}$ & $6.2 \pm 1.2$ & $5.0 \pm 0.7$ & $232756.71201+084644.1368$ & 0.24 & $8.2 \times 4.6$ & \\
\hline (tapered) & $5.2 \pm 1.0$ & & & & & \\
\hline MERLIN, & & & & & & \\
\hline SE & $18.0 \pm 2.3$ & $16.2 \pm 1.1$ & $\begin{array}{lll}0.0 & 0.0\end{array}$ & 0.31 & $82.2 \times 36.8$ & \\
\hline (tapered) & $18.7 \pm 2.4$ & & & & & \\
\hline NW & $3.8 \pm 0.7$ & $3.9 \pm 0.5$ & -452.5 mas 229.5 mas & & & \\
\hline (tapered) & $6.9 \pm 1.1$ & & & & & \\
\hline
\end{tabular}


Table 2. continued.

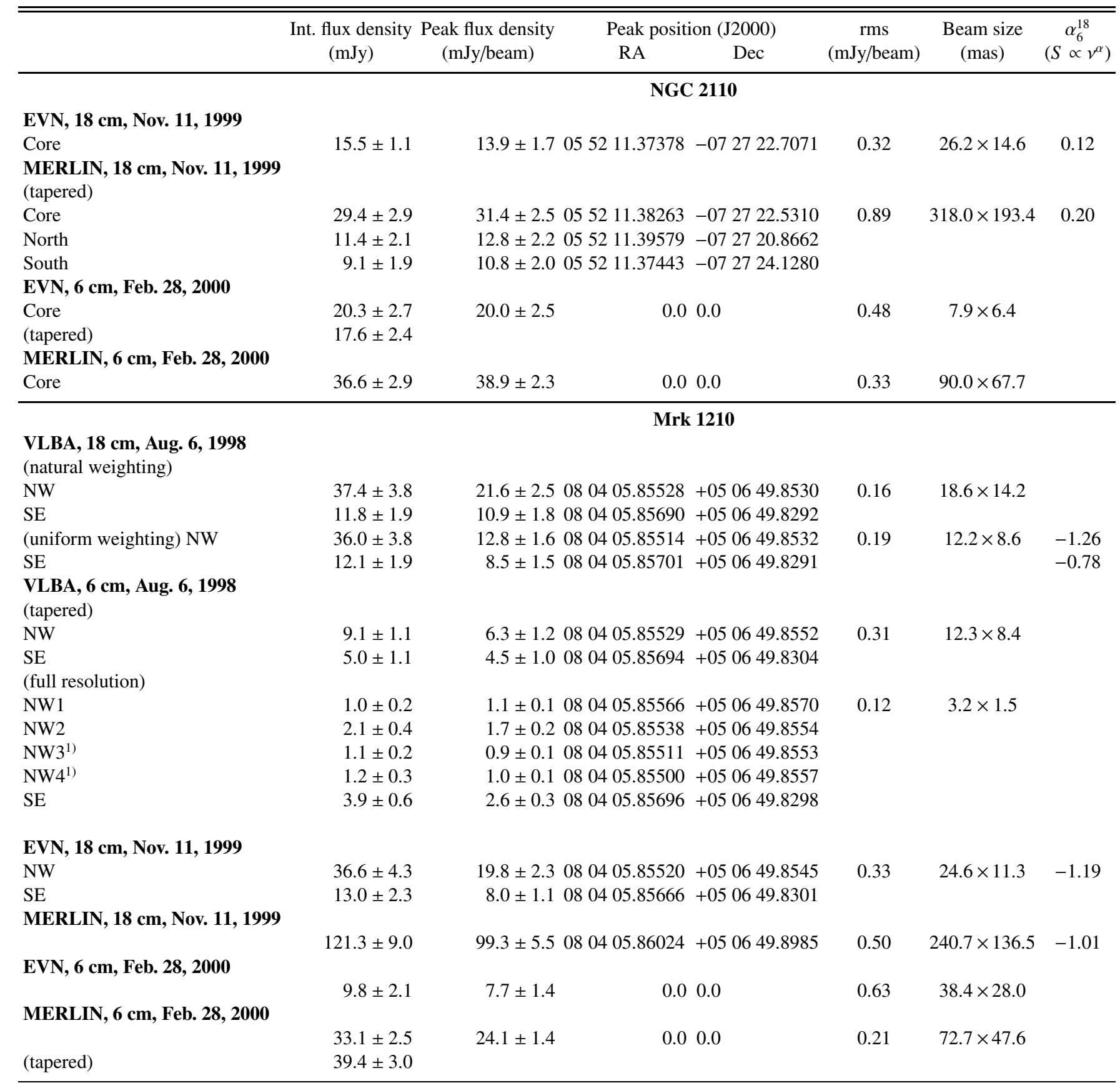

1) Positions and flux densities measured by fitting Gaussians to the image, instead of fitting a quadratic surface and integrating over the component region.

than the MERLIN $6 \mathrm{~cm}$ beam size, almost all of which appears in the VLA image of Colbert et al. (1996).

The VLBI $18 \mathrm{~cm}$ and $6 \mathrm{~cm}$ images in Fig. 1 show three components which we name B0 through B2, with separations of $3.5 \mathrm{pc}(\mathrm{B} 0-\mathrm{B} 1)$ and $5.1 \mathrm{pc}(\mathrm{B} 0-\mathrm{B} 2)$. These three components are unresolved or slightly resolved by the VLBA and EVN. The total $18 \mathrm{~cm}$ flux density recovered by the EVN in 1999.86 was $65 \mathrm{mJy}$, one third of the $6 \mathrm{~cm}$ single-dish emission.

We have aligned images from all four epochs using the centre of $\mathrm{B} 0$ as a reference point to illustrate the relative motions between components inside NGC 5506 (Fig. 2). From 1994.16 to 1997.10 , component B1 has moved $(1.60 \pm 1.26)$ mas ( $1 \sigma$ error), yielding $(0.21 \pm 0.17) c$. However, the error is large, and motion as fast as $0.50 c$ would be required for a $3 \sigma$ detection. After 1997.10, the motion of B1 is uncertain. The position angle in which $\mathrm{B} 1$ might be moving is $(18 \pm 2)^{\circ}$, different from the PA of $(72 \pm 2)^{\circ}$ of $\mathrm{B} 1$ with respect to $\mathrm{B} 0$, so if the motion of $\mathrm{B} 1$ is real, it is not simply radially away from B0. Component B2 showed no consistent believable displacements; the apparent motions of B2 are probably due to limited image fidelity at low SNR. 


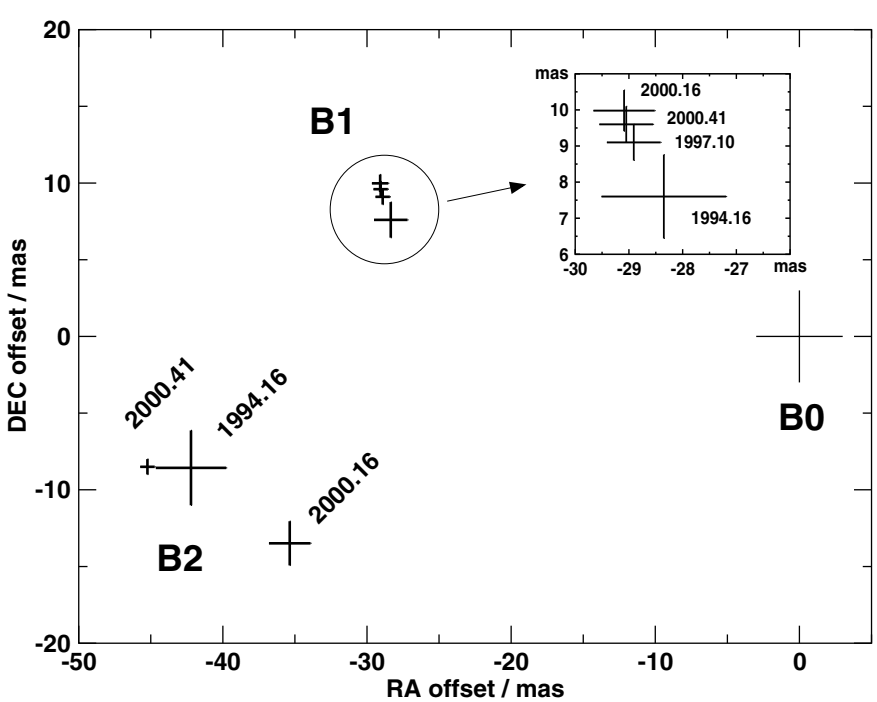

Fig. 2. Diagram illustrating the apparent motions in NGC 5506. The peak positions of $\mathrm{B} 1$ and $\mathrm{B} 2$ from $6 \mathrm{~cm}$ global VLBI, EVN and VLBA are plotted relative to B0. Errors are $1 \sigma$.

The diameters of components B0 and B1 were measured by fitting a Gaussian to the images, and the intrinsic size was determined by deconvolving the beam from the fitted Gaussian. Component B0 proved to be only slightly extended (3.2 mas $\times 2.6$ mas) at $6 \mathrm{~cm}$ compared to the beam size of 5.7 mas $\times 3.7$ mas (VLBA, February 1997), consistent with the near equality of peak and integrated flux densities. Component B1 was significantly resolved in the same observation $(12.5$ mas $\times 8.9$ mas $)$, confirmed by the significantly smaller peak flux density $\left(4.3 \mathrm{mJy} \mathrm{beam}^{-1}\right)$ compared to the integrated flux density ( $24.4 \mathrm{mJy})$. The brightness temperature of component B0, derived from the integrated flux density and deconvolved component size, is $3.6 \times 10^{8} \mathrm{~K}$, whereas the brightness temperature of the resolved component $\mathrm{B} 1$, derived from the peak flux density and beam size, is $1.4 \times 10^{7} \mathrm{~K}$.

Spectral indices were measured using EVN $18 \mathrm{~cm}$ and EVN $6 \mathrm{~cm}$ images tapered to matching resolution. The strongest component (B0) was found to have a flat spectrum ( $\alpha=-0.03$ ) while the other components had steeper spectra ( $\alpha=-0.77$ and -0.3 ).

The spectral index measured for component B0 might be affected by the extended emission to its NW (Fig. 3), adding a negative bias to our measurement. To estimate the size of the effect, we note that the extended emission has a brightness of $2 \mathrm{mJy}_{\text {beam }}^{-1}$ at $6 \mathrm{~cm}$ in a 16.5 mas $\times 8.0$ mas beam, and is present in one quadrant from B0. This emission would contribute a flux density of $3 \mathrm{mJy}$ in the $26.2 \mathrm{mas} \times 14.1 \mathrm{mas}$ tapered beam area at $18 \mathrm{~cm}$ assuming it has a spectral index of -0.7 and that it would be seen by the EVN $18 \mathrm{~cm}$ array but not by the $6 \mathrm{~cm}$ array. Subtracting $3 \mathrm{mJy}$ from the measured flux density of B0 at $18 \mathrm{~cm}$ of 32.9 mJy yields a corrected spectral index of +0.06 , as listed in Table 2 .

Likewise, the spectral index estimated for component B1 might be affected by a possible bridge of faint emission between $\mathrm{B} 1$ and $\mathrm{B} 0$ visible in the combined EVN and MERLIN image in Fig. 3. The emission has a brightness of $2 \mathrm{mJy} \mathrm{beam}^{-1}$

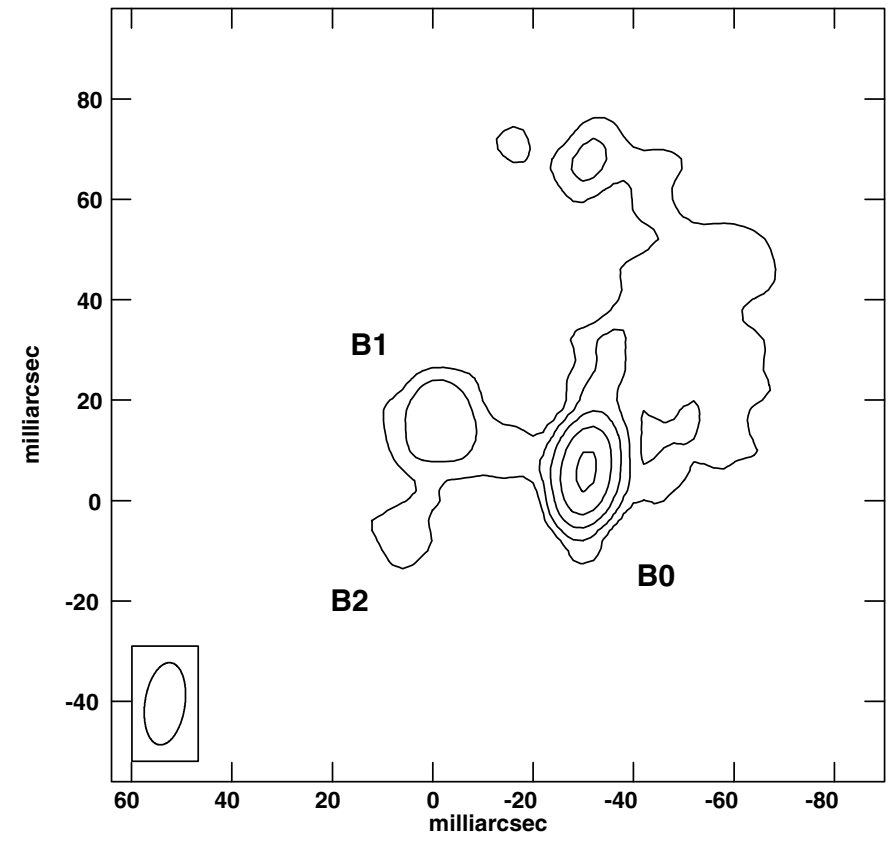

Fig. 3. Combined EVN and MERLIN $6 \mathrm{~cm}$ image of NGC 5506. Offsets are relative to RA 14:13:14.87953, Dec -03:12:27.6799 (J2000), peak flux density is $37.3 \mathrm{mJy} / \mathrm{beam}$, contours are $1 \mathrm{mJy} / \mathrm{beam} \times(2,4,8,16,32)$, beam size is 16.5 mas $\times$ 8.0 mas and natural weighting was used.

at $6 \mathrm{~cm}$ and occupies one octant. This emission would contribute $1.5 \mathrm{mJy}$ to the EVN $18 \mathrm{~cm}$ measurement and would be resolved out by the $6 \mathrm{~cm}$ EVN array. Subtracting these $1.5 \mathrm{mJy}$ from the measured flux density of B1 at $18 \mathrm{~cm}$ of $25.6 \mathrm{mJy}$ yields a corrected spectral index of -0.73 (Table 2).

The flux density of component $\mathrm{B} 0$ remained constant to within the uncertainties. Component B1 faded by a factor of two at $6 \mathrm{~cm}$ and changed its spectral index from $\alpha_{6}^{18}=0.0$ in 1997 to $\alpha_{6}^{18}=-0.77$ in 2000 . The change could be due to an expanding synchrotron source that was initially compact enough to be synchrotron self-absorbed, or else could be due to changing foreground free-free absorption.

Combining the EVN and MERLIN $6 \mathrm{~cm}$ data yields a possible bridge of emission connecting the two brightest components and the detection of very faint diffuse structure north of component B0 (Fig. 3). This diffuse structure is interesting because it suggests a physical connection between the AGN and the radio emission on kpc scales observed with the VLA in C-configuration by Colbert et al. (1996). The MERLIN $18 \mathrm{~cm}$ beam size of 260 mas $\times 150$ mas includes the three components B0-B2 and the diffuse northern emission ( 80 mas $\times$ 40 mas) within a single beam. Thus, the total MERLIN $18 \mathrm{~cm}$ flux of $113 \mathrm{mJy}$ is probably the combined emission from all the structures seen at $6 \mathrm{~cm}$. This diffuse northern emission is not seen in the EVN image because the EVN is insensitive to structures larger than $\approx 35$ mas at $18 \mathrm{~cm}$ or 11 mas at $6 \mathrm{~cm}$. Although component B2 has the same brightness as the northern diffuse region in the MERLIN image, $\mathrm{B} 2$ remains visible in the EVN images whereas the northern diffuse emission does not, because B2 is more compact and so is not resolved away at the higher EVN resolution. 


\subsubsection{Discussion}

The nuclear radio emission of NGC 5506 is resolved into three compact, non-colinear core components within the central $5 \mathrm{pc}$, and a diffuse region of $8 \mathrm{pc}$ extent to the north. The two strongest radio components (B0 and B1) are misaligned by $20^{\circ}$ from the galactic plane and almost perpendicular to the kpc radio structure. On the hundred pc to kpc scale, the radio jet axes in Seyferts have been found to be randomly oriented with respect to the galactic disc rotation axes (e.g. Pringle et al. 1999; Nagar \& Wilson 1999; Kinney et al. 2000), whereas by conservation of angular momentum during accretion, one would expect a common axis. Misalignment of pc and kpc-scale radio emission is shown later to be a common phenomenon in Seyfert galaxies (Table 7, Fig. 7b).

The radio emission from component B1 is peculiar. Its $6 \mathrm{~cm}$ integrated flux density decreased from $30.0 \mathrm{mJy}$ in 1994 to $24.4 \mathrm{mJy}$ in 1997 and $10.8 \mathrm{mJy}$ in February 2000, and then increased slightly to $15.3 \mathrm{mJy}$ in May 2000 (this last increase, however, is not significant within $3 \sigma$ ). Thus, the $6 \mathrm{~cm}$ flux density has constantly decreased, producing a corresponding steepening of the spectrum over this six year period from $\alpha=0.0$ in February 1997 to $\alpha=-0.78$ in May 2000. The combination of flat spectrum and low brightness temperature in 1997 $\left(1.4 \times 10^{7} \mathrm{~K}\right)$ cannot be explained by synchrotron self absorption, and is probably due to free-free absorption. The time variability of the spectrum rules out free-free emission because the spectral cutoff of a gas with such a high temperature is in the $\mathrm{X}$-ray regime and radio spectral index variability as the component cools is therefore not expected.

B0 is a candidate for the location of the AGN core due to its flat spectrum $\left(\alpha_{6}^{18}=0.06\right)$ and compactness.

The alternative picture is that the AGN might lie between $\mathrm{B} 0$ and $\mathrm{B} 1$ and then the two bright components might be working surfaces where double-sided jets from the AGN impact the ISM. Low-surface-brightness emission is seen extending from the hot spots to larger scales (the region of emission north of B0, and component B2) and could be plasma that has flowed through the hot spots and is either deflected by the shock, or is rising buoyantly in the pressure gradient in the ISM. However, B0 has a flat radio spectrum, indicative of optically thick instead of optically thin synchrotron emission, whereas working surfaces, like in compact symmetric objects (CSOs), characteristically show steep spectra. Hence, we favour B0 being the core.

The picture of an AGN core lying between two hotspots seems to describe the radio observations of NGC 7674 discussed in the next section and in Momjian et al. (2003), and of NGC 3079 (Middelberg et al., in prep.).

Ballistic motion of components, when back-extrapolated, should converge on the core. However, in NGC 5506 the B1 component, if its motion is real, moves almost northwards in PA $(18 \pm 2)^{\circ}$, whereas its position with respect to the likely core component $\mathrm{B} 0$ is in $\mathrm{PA}(72 \pm 2)^{\circ}$.

A slight variant on this picture would have precession of the accretion disc move the hot spots "sideways" along a "wall" of ISM leaving behind a trail of fading extended plasma to account for the observed proper motions having a tangential component, and for the "trail" of low surface-brightness emission behind each hot spot.

\subsection{NGC 7674}

NGC 7674 is a face-on spiral galaxy with asymmetric arms and a tidal connection to a nearby compact elliptical galaxy (Hutchings 1996). Observations with the VLA and EVN (Unger et al. 1988) reveal a linear triple radio source of $\sim 0.7^{\prime \prime}$ angular extent. The components are $\sim 0.5^{\prime \prime}$ west and $\sim 0.15^{\prime \prime}$ east of the main peak. Momjian et al. (2003) made a very sensitive observation of NGC 7674 with the VLBA, phased VLA and Arecibo at $1.4 \mathrm{GHz}$ showing the triple source found by Unger et al. (1988) and additional low surfacebrightness emission forming an S-shaped structure.

\subsubsection{Results}

Our MERLIN $18 \mathrm{~cm}$ image (Fig. 4a) shows the well-known triple structure consistent with the VLA A-array $15 \mathrm{GHz}$ image by Unger et al. (1988), but the eastern component was not detected by the EVN or by MERLIN at $6 \mathrm{~cm}$.

The integrated MERLIN $6 \mathrm{~cm}$ flux density was $25.6 \mathrm{mJy}$, lower than the total $6 \mathrm{~cm}$ single-dish flux density of $73 \mathrm{mJy}$ in a $2.4^{\prime}$ beam. The latter was measured three months earlier at Effelsberg in a related project to look for Seyfert variability. Thus, only $35 \%$ of the total emission was detected by the MERLIN observation; the remaining $65 \%$ is also not seen in the VLA $15 \mathrm{GHz}$ image of Unger et al. (1988). Both MERLIN and VLA observations are insensitive to structures larger than 0.15 arcsec, or $90 \mathrm{pc}$.

Our $18 \mathrm{~cm}$ EVN image from November 11, 1999, in Fig. 4b (lower panel) displays a bright, slightly resolved component and point-like radio emission towards the north west, consistent with the structure seen previously by Unger et al. (1988). The SE component is slightly extended in the direction of the NW component.

From the 1985 EVN archive observation we obtained a good-quality $18 \mathrm{~cm}$ image (Fig. 4b, upper panel) with a $49 \sigma$ detection of the SE component and a $9 \sigma$ detection of the NW component. We produced images from the 1985 and the 1999 data sets with matching $(u, v)$ coverages and equal restoring beams $\left(41.9\right.$ mas $\times 24.5$ mas in PA $\left.-29.20^{\circ}\right)$ to look for a change in separation of the components. From the 1985 image, we measured a separation of $(508.7 \pm 0.4)$ mas $(1 \sigma$ error $)$, and from the 1999 image, we measured $(516.0 \pm 0.4)$ mas $(1 \sigma$ error). Thus, we find that the relative separation between the components has increased by $(7.3 \pm 0.6)$ mas in 14.58 years, corresponding to $0.28 \mathrm{pc} \mathrm{yr}^{-1}$ or $v_{\text {app }}=(0.92 \pm 0.07) c$. The separation increased along PA $110^{\circ}$, in agreement with the PA of the the central and the NW component of $117^{\circ}$. The $18 \mathrm{~cm}$ flux densities of the SE and NW component have increased from $22.3 \mathrm{mJy}$ to $37.6 \mathrm{mJy}$ and from $4.1 \mathrm{mJy}$ to $11.0 \mathrm{mJy}$, respectively, the second epoch being in agreement with Momjian et al. (2003). Amplitude calibration errors could in principle produce a scaling of the apparent flux densities, but probably not by such a large factor as seen here. Furthermore, they 


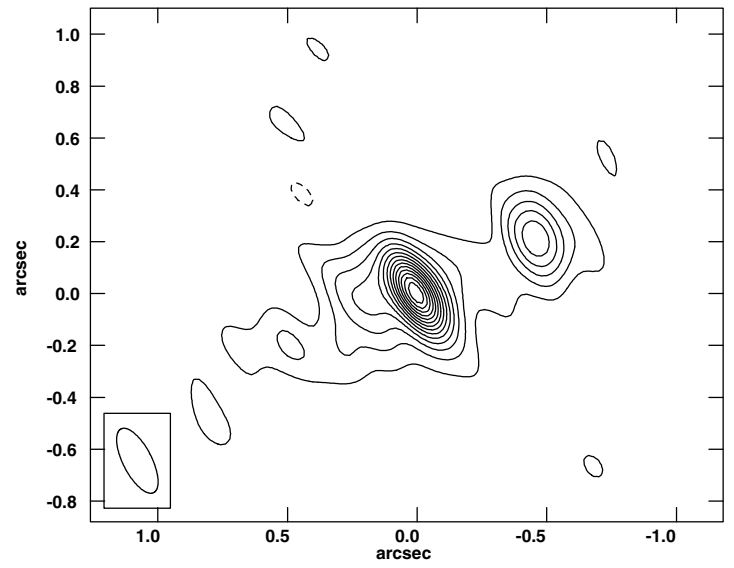

(a) NGC 7674 at $18 \mathrm{~cm}$ (MERLIN). Offsets are relative to RA 23:27:56.71696, Dec 08:46:44.0078 (J2000), peak flux density is $74.9 \mathrm{mJy} / \mathrm{beam}$, contours are $1 \mathrm{mJy} /$ beam $\times(-5,5,10,15,20, \ldots, 75)$, beam size is 276.1 mas $\times 110.4$ mas and uniform weighting was used.

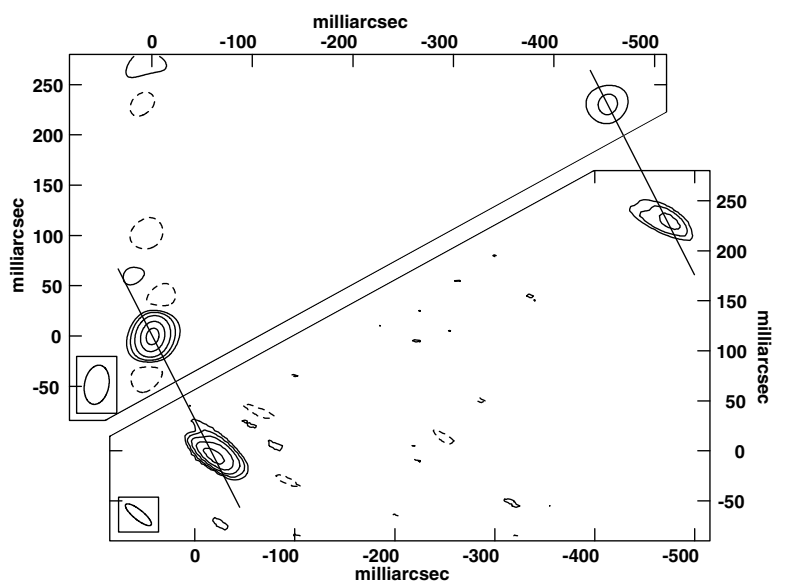

(b) EVN $18 \mathrm{~cm}$ images of NGC 7674. Top: first epoch at 1985.28. Peak flux density is $29.8 \mathrm{mJy} /$ beam, contours are $1 \mathrm{mJy} /$ beam $\times(-2,2,4,8,16,25)$, beam size is 39.3 mas $\times 24.2$ mas and natural weighting was used. Bottom: second epoch at 1999.86. Offsets are relative to RA 23:27:56.71201, Dec 08:46:44:1368 (J2000), peak flux density is $23.9 \mathrm{mJy} / \mathrm{beam}$, contours are $1 \mathrm{mJy} /$ beam $\times(-1,1,2,4,8,16,32)$, beam size is 32.5 mas $\times 9.8$ mas and uniform weighting was used.

Fig. 4. NGC 7674 images.

cannot produce the observed decrease of the ratio of the flux densities from 5.4 to 3.4 . Thus, it is most likely that both components have brightened.

The spectral indices were calculated using the EVN $18 \mathrm{~cm}$ and EVN $6 \mathrm{~cm}$ images tapered to matching resolution. The low surface brightness emission surrounding component NW seen by Momjian et al. (2003) (1.7 $\mu \mathrm{Jy} \mathrm{mas}^{-2}$ ) has a negligibly small effect on the spectral indices measured by us.

\subsubsection{Discussion}

All components in the EVN and MERLIN images of NGC 7674 have steep spectra, and no core identification can be made based on the spectrum. Momjian et al. (2003) suggest that the nucleus might lie between the two components we detected and is free-free absorbed at $21 \mathrm{~cm}$. If so, our observations show that it must also be absorbed at $6 \mathrm{~cm}$.

If the core is indeed located between the two brightest components, then the structure would be reminiscent of that in compact symmetric objects, and the components could be working surfaces where the jets impact the ISM. Then the proper motions would measure the hot-spot advance speed. From the proper motion of $0.28 \mathrm{pc} \mathrm{yr}^{-1}$ and the component separation of $282 \mathrm{pc}$ we derive a dynamical age for the source of $1000 \mathrm{yr}$, which lies within the range of ages measured for CSOs by Polatidis et al. (2002). However, the $18 \mathrm{~cm}$ radio luminosity of the two components in NGC 7674 combined is $8 \times 10^{22} \mathrm{~W} \mathrm{~Hz}^{-1}$, which is three orders of magnitude less than the typical CSOs studied by Polatidis et al. (2002). The high radio luminosity of CSOs is probably a selection effect, since the CSO samples are drawn from flux-density-limited surveys and so automatically exclude lower-luminosity sources. Thus, we may here be observing the low-luminosity end of the CSO phenomenon.

The speed of $(0.92 \pm 0.08) c$ with which the two components separate is, after III Zw 2, the highest found in a Seyfert galaxy. If the components are shocks across jets, then the flow speed of the jet could be even higher. Furthermore, the PA in which the separation increases is in agreement with the relative PA of the central and the NW component, indicating a linear expansion of the source. The separation of $282 \mathrm{pc}$ between the components is comparable to the longest jets found in Seyferts (e.g. NGC 4151, Pedlar et al. 1993 or Mrk 3, Kukula et al. 1993), and shows that jets in Seyfert galaxies can propagate large distances while maintaining high speed.

NGC 7674 is remarkable for its broad asymmetric forbidden line profiles which show highly blue-shifted wings (2000 $\mathrm{km} \mathrm{s}^{-1}$, Unger et al. 1988). These blue wings lie well outside the galaxy rotation curve and a connection to the AGN or jet seems likely (Unger et al. 1988), as has been found in other objects (e.g. Whittle et al. 1988; Axon et al. 1998). Whittle (1992) investigated the acceleration mechanisms of NLR gas in Seyfert galaxies. In his sample, Seyferts such as NGC 7674 with linear radio morphology and radio powers exceeding $10^{22.5} \mathrm{~W} \mathrm{~Hz}^{-1}$ (NGC 7674 has $2.5 \times$ $10^{23} \mathrm{~W} \mathrm{~Hz}^{-1}$, derived from MERLIN $18 \mathrm{~cm}$ integrated flux density) have systematically broader [O III] lines, which he attributes to jet material acting on the NLR gas.

\subsection{NGC 2110}

NGC 2110 is an elliptical or S0 galaxy. VLA observations at $6 \mathrm{~cm}$ and $20 \mathrm{~cm}$ with $0.4^{\prime \prime}$ and $1.2^{\prime \prime}$ resolution by Ulvestad \& Wilson (1983) show a symmetric, S-shaped radio source $\sim 4^{\prime \prime}(600 \mathrm{pc})$ in extent which was interpreted as a twosided, continuous jet interacting with the ISM. This structure 


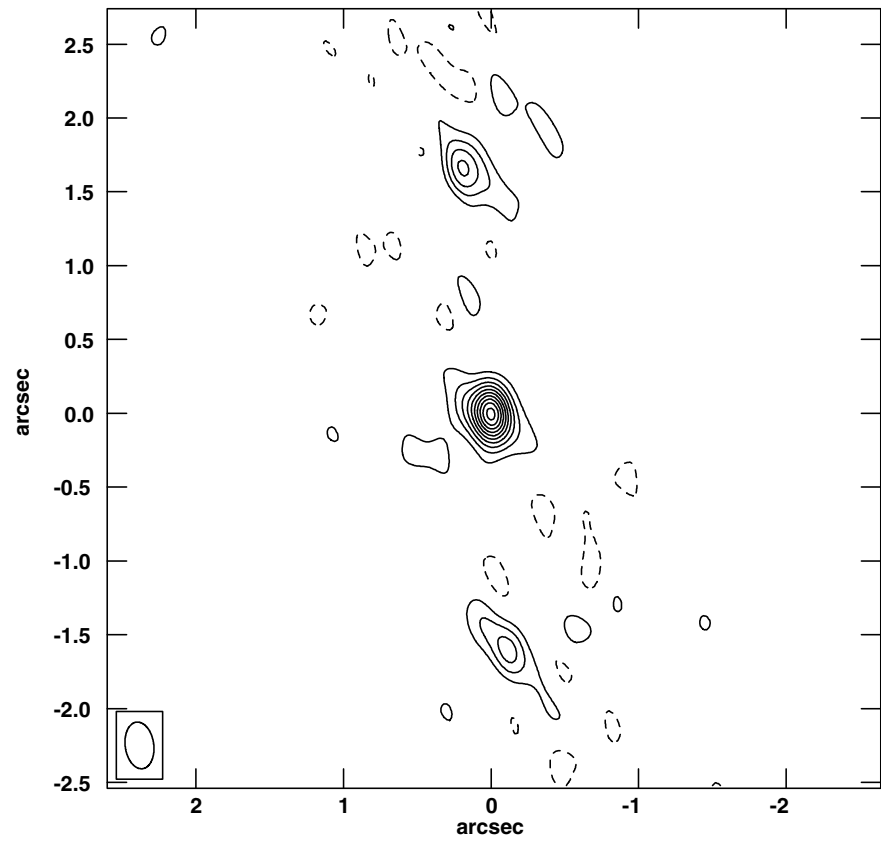

Fig. 5. NGC 2110 at $18 \mathrm{~cm}$ (MERLIN). Offsets are relative to RA 05:52:11.38254, Dec -07:27:22.5262 (J2000), peak flux density is $31.4 \mathrm{mJy} /$ beam, contours are $1 \mathrm{mJy} /$ beam $\times(-3,3,6,9, \ldots, 30)$, beam size is 318.0 mas $\times 193.4$ mas and natural weighting was used. The $(u, v)$ range was $80 \mathrm{k} \lambda$ to $1000 \mathrm{k} \lambda$.

was confirmed in a VLA $3.6 \mathrm{~cm}$ image by Nagar \& Wilson (1999) which also resolved finer details in the jets.

\subsubsection{Results}

Our MERLIN $18 \mathrm{~cm}$ image (Fig. 5, tapered to $0.3 \operatorname{arcsec} \times$ 0.2 arcsec resolution) shows the core and two components, to north and south, at distances of $(1.72 \pm 0.02)^{\prime \prime}$ and $(1.62 \pm$ $0.02)^{\prime \prime}$, respectively, from the central component. These separations are consistent with those measured by Ulvestad \& Wilson (1983) for the brightest parts of the radio lobes. The extended structure seen in their observations was not detected by EVN or MERLIN as the surface brightness $(360 \mathrm{~K})$ is below the MERLIN detection threshold $(2000 \mathrm{~K})$, except for the bright hot spots. The MERLIN $6 \mathrm{~cm}$ observations yielded only detection of a point source (not shown).

The total flux density in the MERLIN $6 \mathrm{~cm}$ image was $37 \mathrm{mJy}$, compared to the single-dish $6 \mathrm{~cm}$ flux density of $154 \mathrm{mJy}$ in a $2.4^{\prime}$ beam measured simultaneously at Effelsberg. Thus, three quarters of the total flux density originates from emission extended over more than $0.7^{\prime \prime}$ or $75 \mathrm{pc}$. The total VLA $6 \mathrm{~cm}$ flux density was $(175 \pm 8) \mathrm{mJy}$ in 1983 with a $0.4^{\prime \prime}$ beam (Ulvestad \& Wilson 1983), which is marginally more than the single-dish flux density in 2000 .

The full-resolution NGC 2110 EVN images at $18 \mathrm{~cm}$ and $6 \mathrm{~cm}$ (not shown) display only an unresolved point source at the position of the VLA observed core. The spectral index of the core was measured using an EVN $6 \mathrm{~cm}$ image tapered to a resolution matching that of the EVN $18 \mathrm{~cm}$ image and the spectrum was found to be rising slightly with increasing frequency $(\alpha=+0.12)$. The MERLIN spectral index of the core $(+0.20)$ was calculated using the full $(u, v)$ range because tapered $6 \mathrm{~cm}$ images had too little $(u, v)$ coverage remaining to yield reliable images, and no detection was made of the north and south components.

Since there is no extended emission on the 11 mas to 35 mas scale near the core, we believe that these spectral indices are unbiased by the short-baseline-mismatch between the arrays. Observations at higher resolution carried out by Mundell et al. (2000) with the VLBA at $3.6 \mathrm{~cm}$ confirm that the core is unresolved down to a resolution of 2 mas $(0.3 \mathrm{pc})$.

\subsubsection{Discussion}

The spectral index of the core in NGC 2110 was estimated by Ulvestad \& Wilson (1983) and Nagar et al. (1999) from VLA observations, giving $\alpha_{6}^{20}=0.0$ and $\alpha_{3.6}^{20}=-0.47$, respectively. However, both measurements suffer from the low resolution of the VLA at $20 \mathrm{~cm}$, which does not allow a clean separation of core and lobes. Therefore, our EVN and MERLIN observations have allowed an accurate measurement of the core spectral index for the first time.

\subsection{Mrk 1210}

Mrk 1210 is an almost face-on, amorphous Seyfert 2 galaxy. It has a compact NLR (Falcke et al. 1998), and shows broad emission lines in polarized light, indicating a hidden type 1 AGN (Tran et al. 1992). Mrk 1210 has also been classified as a Wolf-Rayet-Galaxy due to its bright He II $\lambda 4686$ emission (Storchi-Bergmann et al. 1998). Falcke et al. (1998) imaged Mrk 1210 in $\mathrm{H} \alpha$ and [O III] with the HST, to try to resolve an ionization cone, and at $3.6 \mathrm{~cm}$ with the VLA, with resolutions of 100 mas and 200 mas, respectively. In both of those observations Mrk 1210 appears compact though with a marginally significant extension towards the southeast in both their optical and radio observations.

The single-dish $6 \mathrm{~cm}$ flux density was $76 \mathrm{mJy}$ in a $4.2^{\prime}$ beam measured in 1990 (Griffith et al. 1995) at the Parkes 64 m telescope. Mrk 1210 hosts an $\mathrm{H}_{2} \mathrm{O}$ megamaser with an isotropic luminosity of $84 L_{\odot}$ (Braatz et al. 1994) which might indicate the presence of an edge-on circumnuclear molecular disc or torus.

\subsubsection{Results}

The MERLIN $18 \mathrm{~cm}$ image (not shown) displays an unresolved point source with a possible weak halo to the north-west. The MERLIN $6 \mathrm{~cm}$ image (Fig. 6a) reveals a bright, unresolved central component with a weak extension to the north-west, and a third component to the far south-east, which we name F. The total MERLIN $6 \mathrm{~cm}$ flux density was $39 \mathrm{mJy}$ compared to the $6 \mathrm{~cm}$ single-dish flux density of $54 \mathrm{mJy}$ in a $2.4^{\prime}$ beam measured simultaneously at Effelsberg on the same day, and so the MERLIN image has recovered most of the flux density.

The EVN and VLBA $18 \mathrm{~cm}$ images in Figs. $6 \mathrm{~b}$ and $6 \mathrm{c}$ reveal a bright compact object (named NW) and a weaker component towards the south-east (named SE) at a distance 


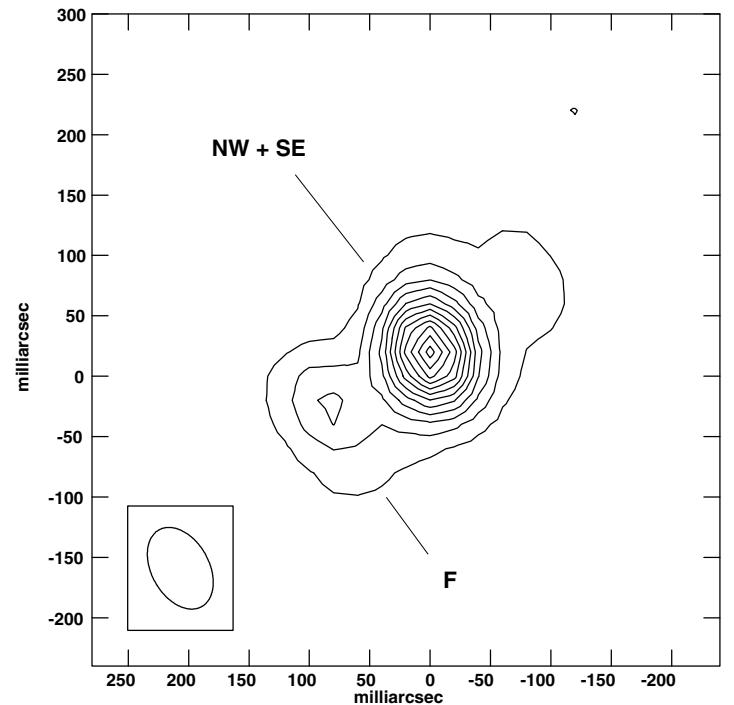

(a) MERLIN $6 \mathrm{~cm}$ image of Mrk 1210. Offsets are relative to RA 08:04:05.86011, Dec 05:06:49.8992 (J2000), peak flux density is $24.1 \mathrm{mJy} / \mathrm{beam}$, contours are $1 \mathrm{mJy} /$ beam $\times(-1,1,3,5,7, \ldots, 23)$, beam size is 72.7 mas $\times 47.6$ mas and uniform weighting was used.

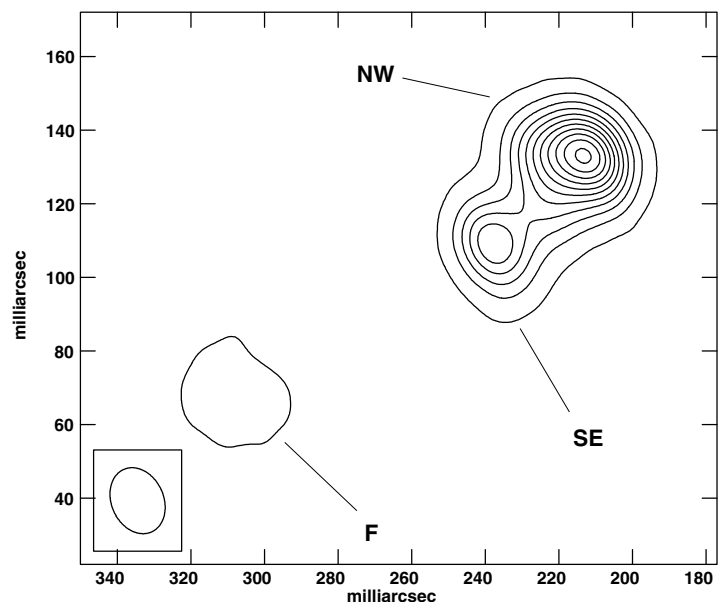

(c) VLBA $18 \mathrm{~cm}$ image of Mrk 1210. Offsets are relative to RA 08:04:05.841, Dec 05:06:49.72 (J2000), peak flux density is $21.6 \mathrm{mJy} /$ beam, contours are $1 \mathrm{mJy} /$ beam $\times(-1$, $1,3,5, \ldots, 21)$, beam size is 18.6 mas $\times 14.2$ mas and natural weighting was used.

Fig. 6. Mrk 1210 images.

of $(32.7 \pm 1.0)$ mas $(8.6 \mathrm{pc})$ from the NW object. We marginally detect a third component at a distance of $\approx 117$ mas $(30.6 \mathrm{pc})$ from NW which is spatially coincident with the weak object $F$ in the MERLIN $6 \mathrm{~cm}$ image. This component is not formally significant but is present in both images and so is probably real and could be the continuation of the radio ejecta. The EVN $6 \mathrm{~cm}$ data (not shown) yield only a marginal detection of the strongest point source. The $6 \mathrm{~cm}$ VLBA image (Fig. 6d) resolves the brightest source into an arc of four components and also resolves the bright component, named SE. The total flux density recovered by the full-resolution EVN and VLBA at $18 \mathrm{~cm}$ was $50 \mathrm{mJy}$ and $49 \mathrm{mJy}$, and at $6 \mathrm{~cm}$ was $10 \mathrm{mJy}$ and $9.3 \mathrm{mJy}$, respectively. The total $6 \mathrm{~cm}$ flux density

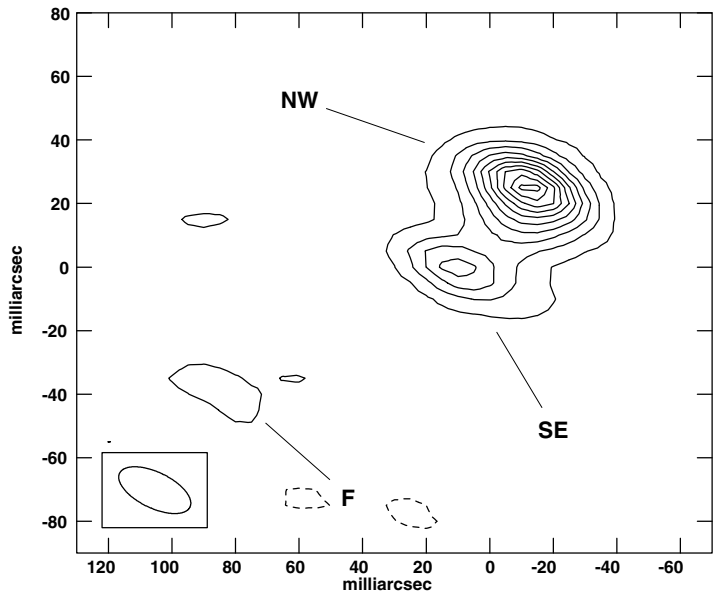

(b) EVN $18 \mathrm{~cm}$ image of Mrk 1210. Offsets are relative to RA 08:04:05.856, Dec 05:06:49.83 (J2000), peak flux density is $19.8 \mathrm{mJy} /$ beam, contours are $1 \mathrm{mJy} /$ beam $\times(-1$, $1,3,5, \ldots, 19)$, beam size is 24.6 mas $\times 11.3$ mas and uniform weighting was used.

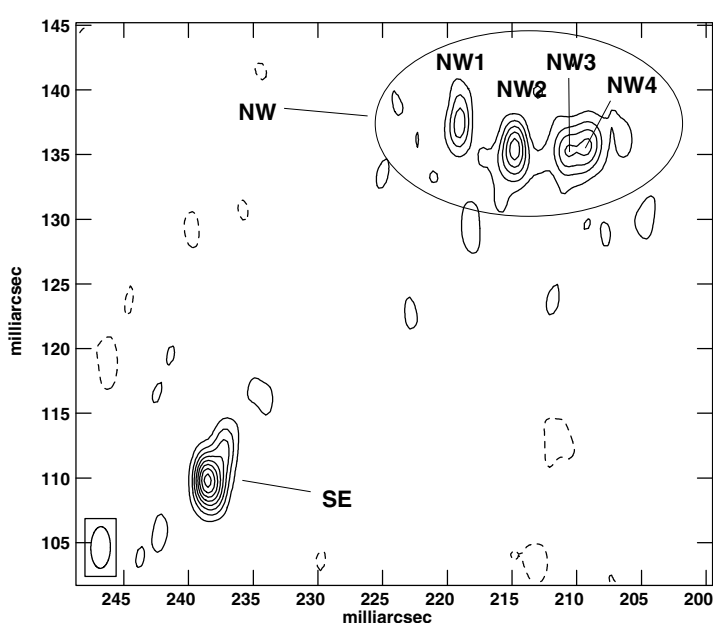

(d) VLBA $6 \mathrm{~cm}$ image of Mrk 1210. Offsets are relative to RA 08:04:05.841, Dec 05:06:49.72 (J2000), peak flux density is $2.5 \mathrm{mJy} /$ beam, contours are $0.1 \mathrm{mJy} /$ beam $\times(-3$, $3,6, \ldots, 24)$, beam size is 3.2 mas $\times 1.5$ mas and uniform weighting was used.

measured by the VLBA in a beam tapered to the $18 \mathrm{~cm}$ resolution is $14 \mathrm{mJy}$, which is still only $35 \%$ of the total MERLIN flux density. So most of the galaxy's $6 \mathrm{~cm}$ emission comes from scales between $3 \mathrm{pc}$ (the largest angular scale to which the EVN is sensitive) and MERLIN's beam size of $16 \mathrm{pc}$, and only little from even larger scales.

Both the NW and SE components have steep spectra, with $\alpha=-1.26$ and $\alpha=-0.78$ in our VLBA images. In our EVN images, the NW component has $\alpha=-1.19$ provided that the weak detection in our EVN $6 \mathrm{~cm}$ observations is the NW component. Since all components have steep spectral indices, the core identification is best done based on proper motions. Our observations here provide the first epoch absolute 
positions and a second epoch should follow after at least five years to allow a reliable detection of proper motion.

The position angle of the SE component with respect to the NW component $\left(122^{\circ} \pm 5^{\circ}\right)$ is perpendicular to the optical polarization vector (PA $29^{\circ}$ ) measured by Tran (1995), as is generally the case in Seyfert $2 \mathrm{~s}$.

\subsubsection{Discussion}

We suggest three scenarios for the radio structure in Mrk 1210.

A free-free emitting disc? The component configuration reminds us of NGC 1068 in which the core component S1 at the jet base is resolved into a 15 mas $(1 \mathrm{pc})$-long structure perpendicular to the jet axis in the sensitive $3.6 \mathrm{~cm}$ VLBA observation by Gallimore et al. (1997). Surrounding this component is an edge-on disc of $\mathrm{H}_{2} \mathrm{O}$ masers whose rotation curve implies a compact central mass of $1.5 \times 10^{7} M_{\odot}$ (Greenhill \& Gwinn 1997). In that case, the $3.6 \mathrm{~cm}$ continuum emission was identified as free-free emission from the ionized inner edge of the torus, based on its flat spectrum, relatively large extent, orientation perpendicular to the jet, and low brightness temperature $\left(10^{6} \mathrm{~K}\right)$, the last ruling out synchrotron self-absorption as the cause of the flat spectrum. In Mrk 1210, the bright arc of four components suggests an edge-on disc, whose existence is further supported by the presence of nuclear water maser emission (although the precise location of the masers is not known). The integrated radio spectrum of component NW is $\alpha_{6}^{18}=-1.2$ using the EVN and $\alpha_{6}^{18}=-1.3$ using the VLBA. This favours optically thin synchrotron radiation rather than flat-spectrum free-free emission. The brightness temperature measured with the VLBA at $18 \mathrm{~cm}$ is $9.2 \times 10^{7} \mathrm{~K}$ and with the EVN at $18 \mathrm{~cm}$ is $7.1 \times 10^{7} \mathrm{~K}$, significantly higher than in NGC 1068 . Thus, although the shape of the radio emitting regions in component NW is suggestive, the structure cannot be free-free emission from the inner edge of a torus like in NGC 1068.

Synchrotron emission from a torus or extended accretion disc? The arc of four components in the NW component could be synchrotron emission from an edge-on viewed torus or accretion disc. However, accretion discs are normally thought to be much smaller and neither accretion discs nor tori are known as synchrotron emitters.

A core and outflow? Perhaps component SE hosts the nucleus. The resolved arc of four components in NW then could be bright knots due to a shock in an extended, low brightness temperature radio outflow, with similar conditions to those in NGC 5506, NGC 1068 or Mrk 231. But both the SE and the F component lack a flat spectrum that would indicate the nucleus.

Further clues might come from absolute proper motion measurements in the future, or from VLBI imaging of the water maser emission.

\section{Statistics}

The range of radio properties displayed by Seyfert galaxies has been explored extensively based on WSRT, VLA or ATCA observations at $20 \mathrm{~cm}, 6 \mathrm{~cm}$ or $3.6 \mathrm{~cm}$ at arcsec resolution by de Bruyn \& Wilson (1978), Ulvestad \& Wilson (1984, 1989),
Unger et al. (1987), Giuricin et al. (1990), Rush et al. (1996), Nagar \& Wilson (1999), Morganti et al. (1999), Ulvestad \& Ho (2001a), and Vir Lal (2001). They studied the range of radio luminosities, radio source sizes, morphologies, alignment between jet and host galaxy, and spectral indices, and looked for dependence of properties on Seyfert type. With the exception of Ulvestad \& Ho (2001a), who found 50\% of low-luminosity Seyferts to have a flat spectrum core, they all found that most Seyfert galaxies have steep, optically-thin synchrotron radio spectra, with $5 \%$ to $35 \%$ (depending on the sample) showing a flat-spectrum core, much less than the rate of occurrence of flat-spectrum cores in powerful radio galaxies. No difference was found between the spectral index distributions of the two Seyfert types.

However, the $0.3^{\prime \prime}$ to several arcsec beams used in those studies do not allow details of nuclear structure to be separated or the distribution of jet bending angles to be constructed. Further, they would probably blend together multiple radio components within the nucleus and could hide the presence of more absorbed cores. Our VLBI observations provide enough resolution to measure separately the emission from individual sub-pc-scale components, and so can find parsec-scale absorbers more efficiently. However, our sample size is too small for any general conclusions.

Many further VLBI observations of Seyfert galaxies are available in the literature but no statistical summary from those observations has been presented. Thus, we made a literature search for VLBI observations of Seyferts to supplement our sample. The resulting list in Table 3 contains, we believe, all VLBI imaging observations of AGN classified as Seyferts by Véron-Cetty \& Véron (2001), published as of August 2003 with a linear resolution of $<10 \mathrm{pc}$, although it is not impossible that we have inadvertently omitted some works. This sample consists mostly of the nearest and best-known Seyferts. They were often selected for observing because they show bright radio nuclei at arcsecond resolution, and so the sample is not complete in any sense. Large, uniform samples of Seyfert galaxies are being observed with the VLBA by Murray et al. (1999) and Schmitt et al. (2002), but the results are not yet available. Proper motions in Seyferts are being presented by Roy et al. (in prep.) and we confine ourselves here to spectral indices and jet bending.

\subsection{Fraction of flat-spectrum components}

We selected from Table 3 a subsample consisting of the Seyfert galaxies for which dual-frequency VLBI observations have been made, including the four objects presented in this paper, to study the component spectral properties at high resolution. This subsample is listed in Table 4.

Of the 16 objects in the subsample, a majority of $13(81 \%)$ has at least one flat or inverted spectrum VLBI component with $\alpha_{6}^{18}>-0.3$. This fraction is $60 \%$ larger than that measured at lower resolution by Ho \& Ulvestad (2001) for the Palomar Seyferts and four or more times larger than the fraction measured at arcsec resolution for the other samples (Table 5). The difference is highly significant for most samples, having 
Table 3. All Seyferts observed with VLBI. Column 1 lists the names we used, Col. 2 the names used by Véron-Cetty \& Véron (2001), Col. 3 the Seyfert types as described by Véron-Cetty \& Véron (2001), Col. 4 the linear resolution in pc, Col. 5 the instrument and frequency used, and Col. 6 the reference to the publication.

\begin{tabular}{|c|c|c|c|c|c|}
\hline Name & Véron name & Class & $\begin{array}{l}\text { Lin. Res. } \\
(\mathrm{pc})\end{array}$ & Instrument & Ref. \\
\hline $3 \mathrm{C} 287.1$ & & S1 & 5.43 & VLBA $5 \mathrm{GHz}$ & Fomalont et al. (2000) \\
\hline 3 C 390.3 & & S 1.5 & 1.09 & global VLBI $5 \mathrm{GHz}$ & Alef et al. (1996) \\
\hline III Zw 2 & & S 1.2 & 0.260 & VLBA $15 / 43 \mathrm{GHz}$ & Brunthaler et al. (2000) \\
\hline Ark 564 & & - & 0.598 & global VLBI $5 \mathrm{GHz}$ & Vir Lal (2001) \\
\hline IC 5063 & PKS 2048-57 & $\mathrm{S} 1 \mathrm{~h}$ & 3.30 & LBA $2.3 \mathrm{GHz}+\mathrm{LBA} H \mathrm{HI}$ & Oosterloo et al. (2000) \\
\hline MCG 8-11-11 & & $\mathrm{S} 1.5$ & 0.635 & global VLBI $5 \mathrm{GHz}$ & Vir Lal (2001) \\
\hline Mrk 1 & & $\mathrm{~S} 2$ & 6.18 & EVN $1.7 \mathrm{GHz}$ & Kukula et al. (1999) \\
\hline Mrk 1 & & $\mathrm{~S} 2$ & 0.550 & global VLBI $5 \mathrm{GHz}$ & Vir Lal (2001) \\
\hline Mrk 3 & & $\mathrm{~S} 1 \mathrm{~h}$ & 5.24 & EVN $1.7 \mathrm{GHz}$ & Kukula et al. (1999) \\
\hline Mrk 78 & & $\mathrm{~S} 2$ & 1.75 & global VLBI $5 \mathrm{GHz}$ & Vir Lal (2001) \\
\hline Mrk 231 & & S1.0 & 0.343 & VLBA $15 \mathrm{GHz}$ & Ulvestad et al. (1999b) \\
\hline Mrk 231 & & S1.0 & 4.09 & VLBA $1.4 / 2.3 / 4.8 / 8.4 / 15 / 22 \mathrm{GHz}$ & Ulvestad et al. (1999a) \\
\hline Mrk 273 & & $\mathrm{~S} 2$ & 7.32 & VLBA $1.4 \mathrm{GHz}$ & Carilli \& Taylor (2000) \\
\hline Mrk 348 & & $\mathrm{~S} 1 \mathrm{~h}$ & 0.131 & VLBA $15 \mathrm{GHz}$ & Ulvestad et al. (1999b) \\
\hline Mrk 348 & & $\mathrm{~S} 1 \mathrm{~h}$ & 6.41 & EVN $1.4 \mathrm{GHz}$ & Neff \& de Bruyn (1983) \\
\hline Mrk 348 & & $\mathrm{~S} 1 \mathrm{~h}$ & 1.49 & VLBA $1.7 / 5.0 / 8 / 15 / 22 \mathrm{GHz}$ & Peck et al. (2003) \\
\hline Mrk 477 & & $\mathrm{~S} 1 \mathrm{~h}$ & 2.39 & global VLBI $5 \mathrm{GHz}$ & Vir Lal (2001) \\
\hline Mrk 530 & NGC 7603 & $\mathrm{~S} 1.5$ & 1.45 & global VLBI $5 \mathrm{GHz}$ & Vir Lal (2001) \\
\hline Mrk 766 & & $\mathrm{~S} 1.5$ & 0.413 & global VLBI $5 \mathrm{GHz}$ & Vir Lal (2001) \\
\hline Mrk 926 & & $\mathrm{~S} 1.5$ & 0.917 & VLBA $8.4 \mathrm{GHz}$ & Mundell et al. (2000) \\
\hline Mrk 1210 & & $\mathrm{~S} 1 \mathrm{~h}$ & 0.392 & EVN $1.6 / 5 \mathrm{GHz}$ VLBA $1.6 / 5 \mathrm{GHz}$ & This paper \\
\hline Mrk 1218 & & S 1.8 & 0.582 & global VLBI $5 \mathrm{GHz}$ & Vir Lal (2001) \\
\hline NGC 1052 & & $\mathrm{~S} 3 \mathrm{~h}$ & 0.015 & VLBA $5 / 8.4 / 22 / 43 \mathrm{GHz}$ & Kadler et al. (2002) \\
\hline NGC 1052 & & $\mathrm{~S} 3 \mathrm{~h}$ & 0.048 & VLBI 1.4-43.2 & Vermeulen et al. (2003) \\
\hline NGC 1052 & & $\mathrm{~S} 3 \mathrm{~h}$ & 0.038 & VLBA 2.3/8.4/15.4 GHZ & Kameno et al. (2001) \\
\hline NGC 1068 & & $\mathrm{~S} 1 \mathrm{~h}$ & 0.221 & VLBA $1.7,5,15 \mathrm{GHz}$ & Roy et al. (1998) \\
\hline NGC 1068 & & $\mathrm{~S} 1 \mathrm{~h}$ & 2.94 & EVN $1.4 \mathrm{GHz}$ & Ulvestad et al. (1987) \\
\hline NGC 1167 & & S3 & 0.895 & global VLBI $5 \mathrm{GHz}$ & Giovannini et al. (2001) \\
\hline NGC 1167 & & S3 & 0.639 & global VLBI $1.6 \mathrm{GHz}$ & Giovannini et al. (1990) \\
\hline NGC 1275 & & $\mathrm{~S} 1.5$ & 0.416 & VLBA $2.3 / 5 / 8.4 / 15.4 / 22 / 43 \mathrm{GHz}$ & Walker et al. (2000) \\
\hline NGC 2110 & & S1i & 0.151 & VLBA $8.4 \mathrm{GHz}$ & Mundell et al. (2000) \\
\hline NGC 2110 & & S1i & 0.966 & EVN $1.6 / 5 \mathrm{GHz}$ & This paper \\
\hline NGC 2273 & & - & 0.342 & global VLBI $5 \mathrm{GHz}$ & Vir Lal (2001) \\
\hline NGC 2639 & & S3 & 0.233 & global VLBI $5 \mathrm{GHz}$ & Vir Lal (2001) \\
\hline NGC 2639 & & S3 & 0.162 & VLBA $1.6 / 5 / 15 \mathrm{GHz}$ & Wilson et al. (1998) \\
\hline NGC 3079 & & $\mathrm{~S} 2$ & 0.044 & VLBA 5/8/22 GHz & Trotter et al. (1998) \\
\hline NGC 3079 & & $\mathrm{~S} 2$ & 0.054 & global VLBI, 5.0 GHz & Irwin \& Seaquist (1988) \\
\hline NGC 3079 & & $\mathrm{~S} 2$ & 0.042 & global VLBI 1.4/8.4/15/22 & Sawada-Satoh et al. (2000) \\
\hline NGC 3147 & & $\mathrm{~S} 2$ & 0.219 & VLBA $1.6 / 2.3 / 5 / 8.4 \mathrm{GHz}$ & Ulvestad \& Ho (2001b) \\
\hline NGC 3227 & & S1.5 & 3.74 & MERLIN $1.6 / 5 \mathrm{GHz}$ & Mundell et al. (1995) \\
\hline NGC 4151 & & S1.5 & 0.116 & VLBA $1.6,5 \mathrm{GHz}$ & Ulvestad et al. (1998) \\
\hline NGC 4151 & & $\mathrm{~S} 1.5$ & 1.29 & EVN $1.7 \mathrm{GHz}$ & Harrison et al. (1986) \\
\hline NGC 4151 & & $\mathrm{~S} 1.5$ & 4.82 & MERLIN $5 \mathrm{GHz}$ & Pedlar et al. (1993) \\
\hline NGC 4168 & & S1.9 & 0.236 & VLBA $5 \mathrm{GHz}$ & Nagar et al. (2002a) \\
\hline NGC 4203 & & $\mathrm{~S} 3 \mathrm{~b}$ & 0.084 & VLBA $1.6 / 2.3 / 5 / 8.4 \mathrm{GHz}$ & Ulvestad \& Ho (2001b) \\
\hline NGC 4258 & & $\mathrm{~S} 2$ & 0.029 & VLBA $22 \mathrm{GHz}$ & Herrnstein et al. (1997) \\
\hline NGC 4258 & & $\mathrm{~S} 2$ & 0.232 & VLBA $1.4 / 1.6 \mathrm{GHz}$ & Cecil et al. (2000) \\
\hline NGC 4395 & & S 1.8 & 0.221 & VLBA $1.4 \mathrm{GHz}$ & Wrobel et al. (2001) \\
\hline NGC 4565 & & S1.9 & 0.207 & VLBA $5 \mathrm{GHz}$ & Falcke et al. (2000) \\
\hline NGC 4579 & & $\mathrm{~S} 3 \mathrm{~b}$ & 0.118 & VLBA $1.6 / 2.3 / 5 / 8.4 \mathrm{GHz}$ & Ulvestad \& Ho (2001b) \\
\hline NGC 4579 & & $\mathrm{~S} 3 \mathrm{~b}$ & 0.246 & VLBA $5 \mathrm{GHz}$ & Falcke et al. (2000) \\
\hline NGC 5252 & & $\mathrm{~S} 2$ & 0.671 & VLBA $8.4 \mathrm{GHz}$ & Mundell et al. (2000) \\
\hline NGC 5506 & & S1i & 0.815 & EVN $1.6 / 5 \mathrm{GHz}$ & This paper \\
\hline NGC 5548 & & $\mathrm{~S} 1.5$ & 0.366 & VLBA $8.4 \mathrm{GHz}$ & Wrobel (2000) \\
\hline NGC 5793 & & $\mathrm{~S} 2$ & 0.135 & VLBA $1.7,8.4,15,22 \mathrm{GHz}$ & Hagiwara et al. (2001) \\
\hline NGC 5793 & & $\mathrm{~S} 2$ & 0.316 & $\mathrm{VLBA}+\mathrm{Y} 27$ 1.6/5 GHz & Hagiwara et al. (2000) \\
\hline
\end{tabular}


Table 3. continued.

\begin{tabular}{|c|c|c|c|c|c|}
\hline Name & Véron name & Class & $\begin{array}{l}\text { Lin. Res. } \\
\text { (pc) }\end{array}$ & Instrument & Ref. \\
\hline NGC 5929 & & S3 & 0.290 & global VLBI $5 \mathrm{GHz}$ & Vir Lal (2001) \\
\hline NGC 5929 & & S3 & 6.44 & MERLIN 0.4/1.6/5 & Su et al. (1996) \\
\hline NGC 7212 & & S1h & 1.37 & global VLBI $5 \mathrm{GHz}$ & Vir Lal (2001) \\
\hline NGC 7469 & & S 1.5 & 0.844 & global VLBI $5 \mathrm{GHz}$ & Vir Lal (2001) \\
\hline NGC 7674 & & $\mathrm{~S} 1 \mathrm{~h}$ & 2.80 & $\mathrm{VLBA}+\mathrm{Y} 27+$ Arecibo $1.4 \mathrm{GHz}$ & Momjian et al. (2003) \\
\hline NGC 7674 & & $\mathrm{~S} 1 \mathrm{~h}$ & 2.58 & EVN $1.6 / 5 \mathrm{GHz}$ & This paper \\
\hline NGC 7674 & & $\mathrm{~S} 1 \mathrm{~h}$ & 2.18 & global VLBI $5 \mathrm{GHz}$ & Vir Lal (2001) \\
\hline NGC 7682 & & $\mathrm{~S} 2$ & 0.996 & global VLBI $5 \mathrm{GHz}$ & Vir Lal (2001) \\
\hline T0109-383 & NGC 424 & $\mathrm{~S} 1 \mathrm{~h}$ & 0.226 & VLBA $8.4 \mathrm{GHz}$ & Mundell et al. (2000) \\
\hline
\end{tabular}

a probability of $<0.1 \%$ of occurring at random. The larger fraction of flat- and inverted-spectrum components found with VLBI is not unexpected since increasing resolution reduces the component blending.

\subsection{Spectral index distributions}

The distribution of $\alpha_{6}^{18}$ from Table 4 is shown in Fig. 7a. The spectral indices lie between -1.8 and +4.6 with a median of -0.40 (using the Kaplan-Meier estimator constructed by ASURV rev 1.2, La Valley et al. 1992 to account for lower limits and discarding upper limits). The distribution shows a tail towards positive spectral index due to four components in NGC 1068, NGC 2639 and NGC 3079 that have strong low-frequency absorption. In the case of two components in NGC 3079, the spectral indices are greater than the maximum $\alpha_{6}^{18}=+2.5$ that can be produced by synchrotron self absorption and so is most likely due to free-free absorption, probably by dense circumnuclear plasma ionized by the AGN.

The tail of extreme positive spectral indices that we see in our sample is not seen at arcsec resolution in the works mentioned above, probably due to component blending. We made a statistical comparison of our VLBI spectral indices to arcsec resolution spectral indices using the Kolmogorov-Smirnov test and chi-squared homogeneity test by treating the lower and upper limits as detections, and by applying survival analysis two-sample tests. The results are summarized in Table 6. Our spectral index distribution was significantly different (i.e. level of significance was less than the critical value of $5 \%$ ) from all the comparison samples according to all tests performed, with the exception of the Palomar Seyferts by Ho \& Ulvestad (2001), which was found to be significantly different in only the chi-squared test. This excess of flat-spectrum sources in the Palomar Seyfert sample was already noticed by Ho \& Ulvestad (2001).

Separating the VLBI measurements by Seyfert type, we found the median $\alpha_{6}^{18}$ for the Sy 1s was -0.75 and for the Sy 2 s was -0.23 , which were not significantly different under the survival analysis two-sample tests $(P=6 \%$ to $12 \%$ that the spectral-index distributions came from the same parent distribution) or under a Kolmogorov-Smirnov test $(P=6.1 \%$ to come from the same parent distribution), treating only lower limits as detections.
Despite the statistical insignificance of a difference between Seyfert types, we notice that the most inverted spectra all occur in objects that have evidence for an absorber. NGC 3079 is a type 2 Seyfert, NGC 1068 and Mrk 348 are type 2 Seyferts with type 1 spectra in polarized light and NGC 2639 shows strong X-ray absorption (Wilson et al. 1998). The inner edge of such an absorber is expected to be ionized by UV emission from the AGN which would produce free-free absorption and hence produce inverted spectra preferentially in the type $2 \mathrm{~s}$. All four objects with inverted radio spectra also host $\mathrm{H}_{2} \mathrm{O}$ masers, which is also consistent with an orientation effect if, for example, the radio core is viewed through a molecular disc or torus, which produces $\mathrm{H}_{2} \mathrm{O}$ maser emission.

\subsection{Jet misalignment}

Kiloparsec-scale linear radio structures in Seyfert galaxies are thought to be energized by jets or outflows originating at the AGN core, and so one might expect pc and kpc-scale radio structures to be aligned. Misalignments could be caused by changes in the ejection axis or by pressure gradients in the ISM. To look for such effects, we compiled kpc-scale radio observations of the VLBI Seyfert sample, where available. The resulting sample of 21 objects in Table 7 is, we believe, a complete list of Seyferts with both pc-scale and kpc-scale observations published, although seven of these objects either have no clear extended structure on one of the scales or the structure was deemed to be affected by processes on scales $>1 \mathrm{kpc}$, e.g., buoyancy forces. In one object, NGC 1167, the double-sided kpc-scale emission is bent, so we quote both. This sample has a large overlap with the sample in Table 4 and, likewise, is not complete in any astronomical sense. Biases such as selection for bright radio nuclei are probably present, but whether they affect the distribution of jet bending angle is unclear. Doppler boosting would favour jets that point towards us, but the jet speeds so far measured in Seyferts are low and boosting effects should be minor.

The distribution of misalignment angles from Table 7 is shown in Fig. 7b. Misalignment in Seyfert jets between pc and kpc-scales is common, with 5 out of 15 objects bending by $45^{\circ}$ or more. The null hypothesis that the jet bending-angle distribution was drawn from a parent population of aligned jets was rejected by a chi-squared test at the $2 \%$ level of significance. 
Table 4. Seyferts from Table 3 that have dual-frequency $(18 \mathrm{~cm}$ and $6 \mathrm{~cm}$ ) VLBI observations with matching beam sizes, from which the spectral index is derived. Asterisks denote objects with at least one component with a flat or inverted spectrum.

\begin{tabular}{|c|c|c|c|c|}
\hline Source & Flat/Inv. & Comp. & $\alpha_{6}^{18}$ & Ref. \\
\hline \multirow[t]{3}{*}{ Mrk 231} & $*$ & $\mathrm{~N}$ & -0.86 & Ulvestad et al. (1999a) \\
\hline & & $\mathrm{C}$ & +0.88 & Ulvestad et al. (1999a) \\
\hline & & $\mathrm{S}$ & -1.54 & Ulvestad et al. (1999a) \\
\hline Mrk 348 & $*$ & & +0.93 & Barvainis \& Lonsdale (1998) \\
\hline \multirow[t]{5}{*}{ Mrk 463E } & & $\mathrm{L}$ & -1.06 & Norris et al., unpublished \\
\hline & & $\mathrm{R}$ & -0.62 & Norris et al., unpublished \\
\hline & & 1 & -0.67 & Norris et al., unpublished \\
\hline & & 2 & -0.77 & Norris et al., unpublished \\
\hline & & 3 & -1.00 & Norris et al., unpublished \\
\hline \multirow[t]{2}{*}{ Mrk 1210} & & NW & -1.26 & This paper \\
\hline & & SE & -0.78 & This paper \\
\hline \multirow[t]{4}{*}{ NGC 1068} & * & $\mathrm{NE}$ & -1.5 & Roy et al. (1998) \\
\hline & & $\mathrm{C}$ & $>2.2$ & Roy et al. (1998) \\
\hline & & $\mathrm{S} 1$ & $>0.6$ & Roy et al. (1998) \\
\hline & & $\mathrm{S} 2$ & $<-1.9$ & Roy et al. (1998) \\
\hline NGC 2110 & $*$ & $\mathrm{C}$ & +0.12 & This paper \\
\hline NGC 2639 & $*$ & & +1.78 & Wilson et al. (1998) \\
\hline \multirow[t]{3}{*}{ NGC 3079} & $*$ & $\mathrm{E}$ & +0.77 & Middelberg et al., in prep. \\
\hline & & A & $>+4.54$ & Middelberg et al., in prep. \\
\hline & & $\mathrm{B}$ & $>+4.36$ & Middelberg et al., in prep. \\
\hline NGC 3147 & $*$ & & +0.20 & Ulvestad \& Ho (2001b) \\
\hline \multirow[t]{3}{*}{ NGC 4151} & $*$ & $\mathrm{E}$ & -0.5 & Ulvestad et al. (1998) \\
\hline & & $\mathrm{D}$ & $<-0.2$ & Ulvestad et al. (1998) \\
\hline & & $\mathrm{F}$ & $<-1.3$ & Ulvestad et al. (1998) \\
\hline NGC 4203 & $*$ & & +0.38 & Ulvestad \& Ho (2001b) \\
\hline NGC 4579 & $*$ & & +0.20 & Ulvestad \& Ho (2001b) \\
\hline \multirow[t]{3}{*}{ NGC 5506} & $*$ & B0 & +0.06 & This paper \\
\hline & & B1 & -0.73 & This paper \\
\hline & & B2 & -0.30 & This paper \\
\hline \multirow[t]{5}{*}{ NGC 5793} & * & $\mathrm{C} 1 \mathrm{C}$ & -0.70 & Hagiwara et al. (2000) \\
\hline & & C1NE & -1.00 & Hagiwara et al. (2000) \\
\hline & & $\mathrm{C} 2 \mathrm{C}$ & -0.46 & Hagiwara et al. (2000) \\
\hline & & $\mathrm{C} 2 \mathrm{~W}$ & -0.72 & Hagiwara et al. (2000) \\
\hline & & $\mathrm{C} 2 \mathrm{E}$ & +0.13 & Hagiwara et al. (2000) \\
\hline \multirow[t]{3}{*}{ NGC 7469} & $*$ & 2 & -0.34 & Norris et al., unpublished \\
\hline & & 3 & +0.09 & Norris et al., unpublished \\
\hline & & 5 & -0.12 & Norris et al., unpublished \\
\hline NGC 7674 & & & -1.78 & This paper \\
\hline
\end{tabular}

The null hypothesis that the distribution was drawn from a uniformly distributed parent population could not be rejected by a chi-squared test, which returned a $25 \%$ level of significance.

We compared the bending angles in Seyferts to those in radio-loud objects using a comparison sample of coredominated radio-loud objects compiled from Table 6 in Pearson \& Readhead (1988), and Table 1 in Conway \& Murphy (1993), which have both VLBI and VLA or MERLIN observations at $5 \mathrm{GHz}$. Those objects show a surprising bimodal distribution of jet bending angle, in which the pc-scale jets tend to align with or to be perpendicular to the kpc-scale structure.
We compared our jet bending angle distribution to the distribution in radio-loud objects using a Kolmogorov-Smirnov test and found no significant difference, the probability of being drawn from the same parent distribution being $14 \%$. However, the Seyfert sample is small and the distribution for the Seyferts was shown in the previous paragraph to be consistent with a uniform distribution.

The frequent misalignments seen in Seyfert galaxy jets could be evidence either for changes in the jet ejection angle and hence of the accretion disc, perhaps by a mechanism such as radiation-driven warping (Pringle 1997), or for 


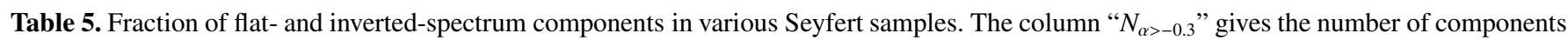
with spectral indices $\alpha>-0.3$ (i.e., components with flat or inverted spectra). The column " $N_{\text {total }}$ " gives the total number of components in each sample. The column $\%$ gives $\left(N_{\alpha>-0.3} / N_{\text {total }}\right) \times 100$. The column $P$ gives the level of significance from a comparison of the fraction of flatand inverted-spectrum components in the VLBI sample of this paper (limits treated as detections), to that of each comparison sample at arcsec resolution, using the difference-of-two-proportions test with Yates correction for continuity (e.g., Glanz 1992).

\begin{tabular}{llrrrr}
\hline \hline Sample & & $N_{\alpha>-0.3}$ & $N_{\text {total }}$ & $\%$ & $P$ \\
\hline This paper & our obs + lit search & 17 & 38 & 45 & \\
Unger et al. (1987) & X-ray Seyferts & 1 & 20 & 5 & $0.3 \%$ \\
Rush et al. (1996) & CfA Seyferts & 3 & 46 & 6.5 & $<0.1 \%$ \\
Rush et al. (1996) & $12 \mu$ m Seyferts & 4 & 40 & 10 & $<0.1 \%$ \\
Morganti et al. (1999) & Distance limited & 13 & 60 & 22 & $1.8 \%$ \\
Ho \& Ulvestad (2001) & Palomar Seyferts & 21 & 45 & 47 & $50 \%$ \\
Ulvestad \& Wilson (1984) & Distance limited & 3 & 40 & 7.5 & $<0.1 \%$ \\
\hline
\end{tabular}

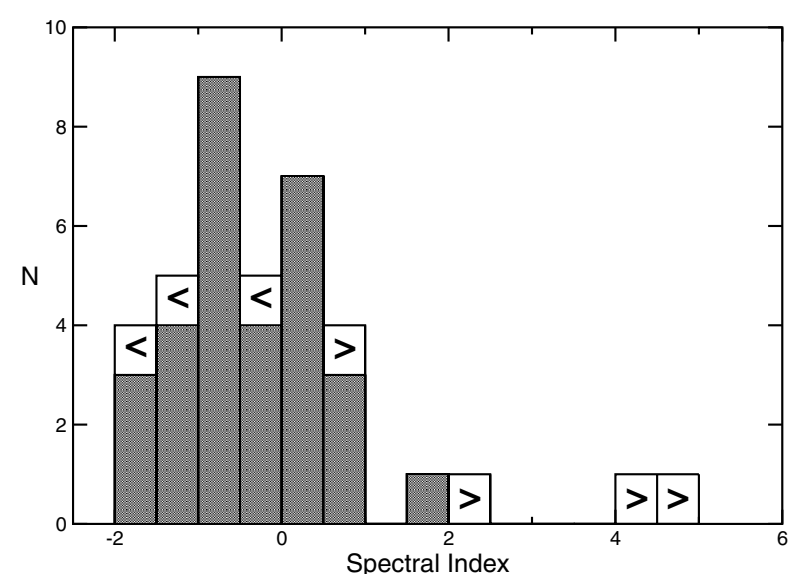

(a)

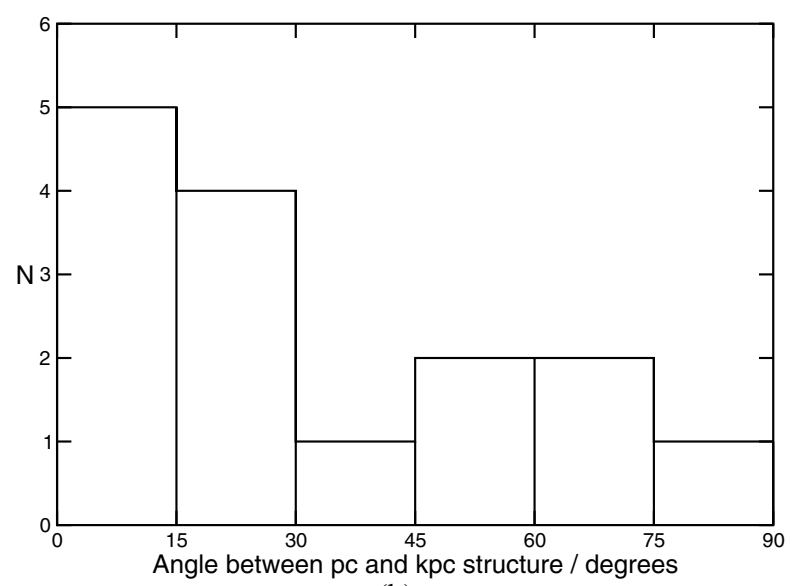

(b)

Fig. 7. Upper panel: histogram of spectral indices between $18 \mathrm{~cm}$ and $6 \mathrm{~cm}$ for the Seyfert sample in Table 4 . Upper and lower limits are indicated by arrows. Lower panel: distribution of angles between pcand kpc-scale structure. For comparison with data from radio galaxies, the data from Table 6 have been reflected onto the range $0^{\circ}$ to $90^{\circ}$.

bending due to pressure gradients in the ISM (collision or buoyancy) through which the jets propagate.

We compared the extended pc-scale radio structures to the host galaxy rotation axes, assuming that the host galaxy is circular and the rotation axis vector is projected onto the galaxy's minor axis, which can be measured from optical images. For all spiral galaxies with pc-scale radio structure, we used the major axes from de Vaucouleurs et al. (1991), subtracted $90^{\circ}$ and reflected the angles onto the range $0^{\circ}$ to $90^{\circ}$. Four objects were not listed in de Vaucouleurs et al. (1991), so we used data from Schmitt \& Kinney (2000) in two cases and we measured the major axes from Hubble Space Telescope archival data in the other two cases. We performed a Spearman rank correlation test and found a correlation coefficient of 0.07 , corresponding to a $82 \%$ significance and so there is no correlation between the directions of the pc-scale structures and the host galaxy rotation axes. However, the sample was small (13 objects) and therefore the test is not strong.

\section{Conclusions}

We have observed the Seyfert galaxies NGC 5506, NGC 7674, NGC 2110 and Mrk 1210 at $18 \mathrm{~cm}$ and $6 \mathrm{~cm}$ with the EVN and MERLIN. NGC 5506 and Mrk 1210 were additionally observed with the VLBA. These observations provided high angular resolution and good surface brightness sensitivity, spectral index measurements, and up to four epochs of observations for proper motion measurements. Our conclusions are as follows.

1. The structure seen on the tens-of-parsec-scale in NGC 7674 shows two bright, steep-spectrum components that might be the working surfaces where double-sided jets ejected from an (unseen) AGN core located between them impact the interstellar medium. Low-surface-brightness emission was seen extending from the hot spots in the direction of VLA-observed emission by Momjian et al. (2003). The diffuse emission could be from material that has flowed through the hotpots and was then either deflected or rose buoyantly in the pressure gradient in the ISM. Alternatively, the bend after the hot spot might be caused by accretion disc precession, which might move the hot spots sideways along a wall of ISM leaving behind a trail of fading extended plasma. These structures are reminiscent of those seen in compact symmetric objects, so we suggest that NGC 7674 is a low-luminosity member of the CSO family.

NGC 5506 shows two bright radio components separated by $3.5 \mathrm{pc}$ with low-surface-brightness emission on larger scales 
Table 6. Comparison of the spectral index distribution of the VLBI Seyfert sample to various other Seyfert samples. The level of significance gives the probability that the spectral index distribution of the comparison sample and the VLBI seyfert sample in this paper were drawn from the same parent population.

\begin{tabular}{|c|c|c|c|c|c|c|}
\hline \multirow[b]{2}{*}{ Sample } & & \multicolumn{5}{|c|}{ Level of Significance } \\
\hline & & $\chi^{2}$ & KS & Logrank & Gehan & Peto-Peto \\
\hline Unger et al. (1987) & X-ray Seyferts & $0.3 \%$ & $0.7 \%$ & $0.3 \%$ & $8.3 \%$ & $8.3 \%$ \\
\hline Rush et al. (1996) & CfA Seyferts & $0.1 \%$ & $0.1 \%$ & $0.0 \%$ & $0.5 \%$ & $0.5 \%$ \\
\hline Rush et al. (1996) & $12 \mu \mathrm{m}$ Seyferts & $0.1 \%$ & $0.6 \%$ & $0.0 \%$ & $2.0 \%$ & $2.0 \%$ \\
\hline Morganti et al. (1999) & Distance Limited & $0.1 \%$ & $0.3 \%$ & $0.0 \%$ & $1.2 \%$ & $1.2 \%$ \\
\hline Ho \& Ulvestad (2001) & Palomar Seyferts & $1.0 \%$ & $27 \%$ & $44 \%$ & $75 \%$ & $77 \%$ \\
\hline Ulvestad \& Wilson (1984) & Distance Limited & $0.1 \%$ & $0.3 \%$ & $0.0 \%$ & $0.7 \%$ & $0.7 \%$ \\
\hline
\end{tabular}

extending towards the VLA-scale emission. The brightest compact component $(\mathrm{B} 0)$ has a flat radio spectrum $(\alpha=0.06)$ and could represent the nucleus.

In NGC 2110 and Mrk 1210, the overall structure is a triple radio source. For NGC 2110, the flat spectrum $(\alpha=0.12)$ and the symmetric structure of the VLA-observed jets and the components at the ends of these jets (also seen in our MERLIN image) show conclusively that the central source represents the nucleus. In Mrk 1210, both the SE and the NW components have steep radio spectra and here the location of the nucleus is unclear.

If jets in Seyferts are initially relativistic and are then disrupted by interaction with the ISM, we might expect significant faint, diffuse radio emission. Such faint emission (brighter than $2 \mathrm{mJy}$ beam $^{-1}$ ) was seen in only NGC 5506.

2. A proper motion of $(0.92 \pm 0.07) c$ was measured in NGC 7674, and a $3 \sigma$ upper limit of $0.50 c$ was found for the motion of B1 in NGC 5506 with respect to B0. From the high speed measured in NGC 7674 between components 282 pc apart we derived a dynamical age of $1000 \mathrm{yr}$. This speed and age is similar to those seen in CSOs. That the components have high speed after propagating hundreds of parsecs shows that the ISM in this object has not stopped the jet very effectively. The speed measured in NGC 7674 is faster than those measured in other Seyferts by Ulvestad et al. (1998), Ulvestad et al. (1999b) and Roy et al. (2000), except for III Zw 2, though is slow compared to the speeds at equivalent scales in powerful radio galaxies. This suggests, but does not prove, that the jets are launched with an intrinsically lower speed than in radio galaxies. Further support comes from the preference of Seyfert nuclei for earlytype spirals, which have relatively low-density ISM even close to the nucleus, and hence it seems unlikely that the jets are significantly slowed.

3. We mentioned in the introduction the many possible reasons for low jet power in Seyferts that have been suggested in the literature. Our observations of proper motions favour an intrinsic over an extrinsic cause since the ISM has not stopped the component in NGC 7674, and no emission from disrupted jets was seen in three of four Seyferts. Any extrinsic deceleration must happen on scales less than $3.5 \mathrm{pc}$. (However, the bends in the radio sources at distances of $3.5 \mathrm{pc}$ and $282 \mathrm{pc}$ in NGC 5506 and NGC 7674 show that the putative jets may nevertheless feel an influence from the NLR.) Of the intrinsic causes mentioned in the introduction, we cannot constrain the role of black-hole spin or magnetic field strength in the accretion disc. The expanding plasmon model of Pedlar et al. (1985) predicts that the radio components expand into an ISM of density $1 \mathrm{~cm}^{-3}$ at speeds initially of $\geq 10^{4} \mathrm{~km} \mathrm{~s}^{-1}$ and would reach a size of $30 \mathrm{pc}$ after $2000 \mathrm{yr}$ by which time the expansion speed would have been reduced to $5000 \mathrm{~km} \mathrm{~s}^{-1}$. At this speed and at the distance of NGC 5506 over the $6.25 \mathrm{yr}$ span of our observations, the component diameters would increase by 0.3 mas, or, in NGC 7674, over 14.58 yr by 0.1 mas. An increase by this amount is, unfortunately, much smaller than could be measured with our data.

4. Spectral indices: we asked in the introduction whether Seyfert nuclei show a single compact synchrotron selfabsorbed core component at base of a collimated jet, like scaled-down radio galaxies. We found flat- or absorbedspectrum components in NGC 5506 and NGC 2110 with brightness temperatures of $\geq 3 \times 10^{7} \mathrm{~K}$. The components in NGC 5506 have no clear axis of symmetry and the flat spectrum could be caused by either free-free absorption or synchrotron self-absorption. The morphology of NGC 2110 shows collimated jets on VLA scales and so is the only object in our sample with a flat-spectrum VLBI core and large-scale jets. Thus, the classical conically-expanding Blandford-Königl jet structure (Blandford \& Konigl 1979, see also Falcke \& Biermann 1995) is sometimes but not often seen in our Seyfert galaxies.

5. Statistics of selected radio properties: to improve the reliability of any conclusions we enlarged our sample by drawing Seyferts from the literature that were observed with VLBI and found that flat- or absorbed- spectrum cores were present in 13 of 16 cases. The most strongly absorbed components tend to be in objects that also show other evidence for absorption, such as water maser emission or large X-ray absorption columns. In those cases, according to the Seyfert unification schemes, the flat-spectrum components would be viewed through an edgeon obscuring disc or torus, the ionized inner edge of which could be causing free-free absorption. If so, the situation in those objects is different from the Blandford-Königl jet model. However the case for free-free absorption is unambiguous in only one galaxy (NGC 3079) for which the spectral index 
Table 7. Subset of sources from Table 3 for which both pc and kpc-scale structures have been observed. The misalignment angle, $\Delta \Theta$, is calculated as $\Theta(\mathrm{pc})-\Theta(\mathrm{kpc})$, and has then been reflected onto the range of $0^{\circ}$ to $90^{\circ}$. For the seven objects at the end of the table, either the $\mathrm{PA}$ information of the kpc scale structure was not completely reliable or was unavailable. The position angles of the galaxy minor axes in the last column were drawn from de Vaucouleurs et al. (1991), except for those marked $a$ taken from Schmitt \& Kinney (2000) and those marked $b$ measured by us from Hubble Space Telescope archival images (see Sect. 4.3).

\begin{tabular}{|c|c|c|c|c|c|c|c|}
\hline Name & $\begin{array}{l}\text { Ref. } \\
\text { (pc) }\end{array}$ & $\begin{array}{c}\Theta(\mathrm{pc}) \\
(\mathrm{deg})\end{array}$ & $\begin{array}{c}\Theta(\mathrm{kpc}) \\
(\mathrm{deg})\end{array}$ & $\begin{array}{c}\Delta \Theta \\
(\operatorname{deg})\end{array}$ & Array & Ref. & $\begin{array}{c}\Theta \text { (opt.) } \\
\text { deg }\end{array}$ \\
\hline \multirow[t]{2}{*}{ 3C 390.3} & 1.09 & 323 & & 0 & global VLBI $5 \mathrm{GHz}$ & Alef et al. (1996) & \\
\hline & 1210 & & 323 & & VLA $5 \mathrm{GHz}$ & Alef et al. (1996) & \\
\hline \multirow[t]{2}{*}{ Mrk 78} & 1.75 & 40 & & 55 & global VLBI $5 \mathrm{GHz}$ & Vir Lal (2001) & \\
\hline & 504 & & 275 & & VLA $5 \mathrm{GHz}$ & Vir Lal (2001) & \\
\hline \multirow[t]{2}{*}{ Mrk 231} & 0.343 & 93 & & 77 & VLBA $15 \mathrm{GHz}$ & Ulvestad et al. (1999b) & 100 \\
\hline & 3270 & & 170 & & VLA $1.5 / 8.4 / 15 \mathrm{GHz}$ & Ulvestad et al. (1999a) & \\
\hline \multirow[t]{2}{*}{ Mrk 1218} & 0.582 & 330 & & 13 & global VLBI $5 \mathrm{GHz}$ & Vir Lal (2001) & $130^{b}$ \\
\hline & 294 & & 317 & & VLA $5 \mathrm{GHz}$ & Vir Lal (2001) & \\
\hline \multirow[t]{2}{*}{ NGC 1052} & 0.015 & 65 & & 30 & VLBA $5 / 8.4 / 22 / 43 \mathrm{GHz}$ & Kadler et al. (2002) & \\
\hline & 162 & & 275 & & MERLIN $1.4 \mathrm{GHz}$ & Kadler et al. (2003) & \\
\hline \multirow[t]{2}{*}{ NGC 1068} & 0.221 & 11 & & 29 & VLBA $1.7,5,15 \mathrm{GHz}$ & Roy et al. (1998) & 160 \\
\hline & 217 & & 40 & & VLA $5 \mathrm{GHz}$ & Ho \& Ulvestad (2001) & \\
\hline \multirow[t]{2}{*}{ NGC 1167} & 0.895 & 140 & 313 & 7 & global VLBI 5 GHz & Giovannini et al. (2001) & \\
\hline & 131 & & 66 & 74 & VLA $5 \mathrm{GHz}$ & Sanghera et al. (1995) & \\
\hline \multirow[t]{2}{*}{ NGC 1275} & 0.416 & 353 & & 23 & VLBA $2.3 / 5 / 8.4 / 15.4 / 22 / 43 \mathrm{GHz}$ & Walker et al. (2000) & \\
\hline & 382 & & 150 & & VLA $1.4 \mathrm{GHz}$ & Pedlar et al. (1990) & \\
\hline \multirow[t]{2}{*}{ NGC 3079} & 0.044 & 125 & & 65 & VLBA 5/8/22 GHz & Trotter et al. (1998) & 75 \\
\hline & 310 & & 60 & & VLA $1.4 / 4.9$ & Duric et al. (1983) & \\
\hline \multirow[t]{2}{*}{ NGC 4151} & 0.116 & 257 & & 3 & VLBA $1.6,5 \mathrm{GHz}$ & Ulvestad et al. (1998) & 140 \\
\hline & 104 & & 80 & & VLA $5 \mathrm{GHz}$ & Ho \& Ulvestad (2001) & \\
\hline \multirow[t]{2}{*}{ NGC 4258} & 0.029 & 3 & & 8 & VLBA $22 \mathrm{GHz}$ & Herrnstein et al. (1997) & 60 \\
\hline & 125 & & 355 & & VLA $1.5 \mathrm{GHz}$ & Cecil et al. (2000) & \\
\hline \multirow[t]{2}{*}{ NGC 7212} & 1.37 & 257 & & 52 & global VLBI $5 \mathrm{GHz}$ & Vir Lal (2001) & $133^{a}$ \\
\hline & 609 & & 25 & & VLA $5 \mathrm{GHz}$ & Vir Lal (2001) & \\
\hline \multirow[t]{2}{*}{ NGC 7469} & 0.844 & 28 & & 35 & global VLBI $5 \mathrm{GHz}$ & Vir Lal (2001) & 35 \\
\hline & 193 & & 353 & & VLA $5 \mathrm{GHz}$ & Vir Lal (2001) & \\
\hline \multirow[t]{2}{*}{ NGC 7674} & 2.80 & 274 & & 22 & VLBA+Y27+Arecibo $1.4 \mathrm{GHz}$ & Momjian et al. (2003) & $64^{a}$ \\
\hline & 178 & & 296 & & MERLIN 1.7 & Momjian et al. (2003) & \\
\hline \multirow[t]{2}{*}{ III Zw 2} & 0.260 & 303 & & 63 & VLBA $15 / 43 \mathrm{GHz}$ & Brunthaler et al. (2000) & \\
\hline & 3460 & & 240 & & VLA $1.5 \mathrm{GHz}$ & Brunthaler, priv. comm. & \\
\hline Ark 564 & 0.598 & 282 & & & global VLBI $5 \mathrm{GHz}$ & Vir Lal (2001) & 28 \\
\hline \multirow[t]{2}{*}{ IC 5063} & 3.30 & 66 & & 49 & LBA $2.3 \mathrm{GHz}+$ LBA HI & Oosterloo et al. (2000) & \\
\hline & 242 & & 115 & & ATCA $8.3 / 1.4 \mathrm{GHz}$ & Morganti et al. (1998) & \\
\hline \multirow[t]{2}{*}{ NGC 2110} & 0.151 & 350 & & 16 & VLBA $8.4 \mathrm{GHz}$ & Mundell et al. (2000) & \\
\hline & 240 & & 186 & & VLA $1.4 / 5 \mathrm{GHz}$ & Ulvestad \& Wilson (1983) & \\
\hline NGC 3147 & 0.219 & 317 & & & VLBA $1.6 / 2.3 / 5 / 8.4 \mathrm{GHz}$ & Ulvestad \& Ho (2001b) & 65 \\
\hline \multirow[t]{2}{*}{ NGC 5506} & 0.815 & 73 & & 76 & EVN $1.6 / 5 \mathrm{GHz}$ & This paper & 1 \\
\hline & 359 & & 177 & & VLA $4.9 \mathrm{GHz}$ & Colbert et al. (1996) & \\
\hline NGC 7682 & 0.996 & 125 & & & global VLBI $5 \mathrm{GHz}$ & Vir Lal (2001) & $68^{b}$ \\
\hline
\end{tabular}

is $>+2.5$. The other 12 Seyferts have $-0.3<\alpha<+2.5$ and so spectral turnovers could be produced by either synchrotron self-absorption or free-free absorption.

Where extended structures are present in the enlarged sample (14 Seyferts), the position angle differences between pc-scale and kpc-scale radio emission were found to be uniformly distributed between $0^{\circ}$ and $90^{\circ}$. Such bends could be due to changes in the jet ejection axis or due to pressure gradients in the ISM. No correlation was found between the axis of pc-scale radio structure and the rotation axis of the host galaxy. 
Acknowledgements. We thank the referee (R. Morganti) for the time and care that she invested in critiquing our manuscript. Her numerous suggestions have improved the paper enormously. We wish to thank R. W. Porcas for providing 15 year old $T_{\text {sys }}$ measurements for the amplitude calibration of the 1985 EVN observations and Jim Ulvestad for help with the reduction of the Mrk 1210 VLBA data. The Effelsberg telescope is operated by the Max-PlanckInstitut für Radioastronomie. The European VLBI Network is a joint facility of European, Chinese and other radio astronomy institutes funded by their national research councils. The VLBA is an instrument of the National Radio Astronomy Observatory, a facility of the National Science Foundation, operated under cooperative agreement by Associated Universities, Inc. This research was supported in part by NASA through grant NAG81755 to the University of Maryland and has made use of the NASA/IPAC Extragalactic Database (NED) which is operated by the Jet Propulsion Laboratory, California Institute of Technology, under contract with the National Aeronautics and Space Administration.

\section{References}

Alef, W., Wu, S. Y., Preuss, E., Kellermann, K. I., \& Qiu, Y. H. 1996, A\&A, 308, 376

Antonucci, R. R. J., \& Miller, J. S. 1985, ApJ, 297, 621

Axon, D. J., Marconi, A., Capetti, A., et al. 1998, ApJ, 496, L75

Barvainis, R., \& Lonsdale, C. 1998, AJ, 115, 885

Bicknell, G. V., Dopita, M. A., Tsvetanov, Z. I., \& Sutherland, R. S. 1998, ApJ, 495, 680

Blandford, R. D., \& Konigl, A. 1979, ApJ, 232, 34

Bower, G., Wilson, A., Morse, J. A., et al. 1995, ApJ, 454, 106

Braatz, J. A., Wilson, A. S., \& Henkel, C. 1994, ApJ, 437, L99

Browne, I. W. A., Wilkinson, P. N., Patnaik, A. R., \& Wrobel, J. M. 1998, MNRAS, 293, 257

Brunthaler, A., Falcke, H., Bower, G. C., et al. 2000, A\&A, 357, L45

Capetti, A., Axon, D. J., \& Macchetto, F. D. 1997, ApJ, 487, 560

Carilli, C. L., \& Taylor, G. B. 2000, ApJ, 532, L95

Cecil, G., Greenhill, L. J., DePree, C. G., et al. 2000, ApJ, 536, 675

Colbert, E. J. M., Baum, S. A., Gallimore, J. F., O'Dea, C. P., \& Christensen, J. A. 1996, ApJ, 467, 551

Conway, J. E., \& Murphy, D. W. 1993, ApJ, 411, 89

de Bruyn, A. G., \& Wilson, A. S. 1978, A\&A, 64, 433

de Vaucouleurs, G., de Vaucouleurs, A., Corwin, H. G., et al. 1991, Third Reference Catalogue of Bright Galaxies, 1-3, XII (Berlin, Heidelberg, New York: Springer-Verlag)

Duric, N., Seaquist, E. R., Crane, P. C., Bignell, R. C., \& Davis, L. E. 1983, ApJ, 273, L11

Falcke, H., \& Biermann, P. L. 1995, A\&A, 293, 665

Falcke, H., Nagar, N. M., Wilson, A. S., \& Ulvestad, J. S. 2000, ApJ, 542, 197

Falcke, H., Wilson, A. S., \& Simpson, C. 1998, ApJ, 502, 199

Fomalont, E. B., Frey, S., Paragi, Z., et al. 2000, ApJS, 131, 95

Gallimore, J. F., Baum, S. A., \& O’Dea, C. P. 1997, Nature, 388, 852

Giovannini, G., Cotton, W. D., Feretti, L., Lara, L., \& Venturi, T. 2001, ApJ, 552, 508

Giovannini, G., Feretti, L., \& Comoretto, G. 1990, ApJ, 358, 159

Giuricin, G., Mardirossian, F., Mezzetti, M., \& Bertotti, G. 1990, ApJS, 72, 551

Glanz, S. A. 1992, Primer of Biostatistics (New York: McGraw-Hill), 3rd ed.

Greenhill, L. J., \& Gwinn, C. R. 1997, Ap\&SS, 248, 261

Griffith, M. R., Wright, A. E., Burke, B. F., \& Ekers, R. D. 1995, ApJS, 97, 347
Hagiwara, Y., Diamond, P. J., Nakai, N., \& Kawabe, R. 2000, A\&A, 360, 49

Hagiwara, Y., Diamond, P. J., Nakai, N., \& Kawabe, R. 2001, ApJ, 560,119

Harrison, B., Pedlar, A., Unger, S. W., et al. 1986, MNRAS, 218, 775

Herrnstein, J. R., Moran, J. M., Greenhill, L. J., et al. 1997, ApJ, 475, L17

Ho, L. C., \& Ulvestad, J. S. 2001, ApJS, 133, 77

Hutchings, J. B. 1996, AJ, 111, 712

Irwin, J. A., \& Seaquist, E. R. 1988, ApJ, 335, 658

Ivezić, Ž., Menou, K., Knapp, G. R., et al. 2002, AJ, 124, 2364

Kadler, M., Ros, E., Kerp, J., et al. 2003, in The Physics of Relativistic Jets in the CHANDRA and XMM Era, ed. G. Brunetti, D. E. Harris, R. M. Sambruna, \& G. Setti

Kadler, M., Ros, E., Lobanov, A. P., \& Falcke, H. 2002, in Proceedings of SRT: the impact of large antennas on Radio Astronomy and Space Science

Kameno, S., Sawada-Satoh, S., Inoue, M., Shen, Z., \& Wajima, K. 2001, PASJ, 53, 169

Keel, W. C. 1996, ApJS, 106, 27

Kellermann, K. I., Sramek, R., Schmidt, M., Shaffer, D. B., \& Green, R. 1989, AJ, 98, 1195

Kinney, A. L., Schmitt, H. R., Clarke, C. J., et al. 2000, ApJ, 537, 152

Kukula, M. J., Ghosh, T., Pedlar, A., \& Schilizzi, R. T. 1999, ApJ, 518,117

Kukula, M. J., Ghosh, T., Pedlar, A., et al. 1993, MNRAS, 264, 893

Laor, A. 2000, ApJ, 543, L111

La Valley, M., Isobe, T., \& Feigelson, E. 1992, in Astronomical Data Analysis Software and Systems I, ASP Conf. Ser., 25, 245

Lestrade, J.-F. 1991, in IAU Col., 131, Radio Interferometry, Theory, Techniques, and Applications, ASP Conf. Ser., 19, 289

Martin, P. G., Thompson, I. B., Maza, J., \& Angel, J. R. P. 1983, ApJ, 266, 470

Meier, D. L., Edgington, S., Godon, P., Payne, D. G., \& Lind, K. R. 1997, Nature, 388, 350

Miller, L., Peacock, J. A., \& Mead, A. R. G. 1990, MNRAS, 244, 207

Momjian, E., Romney, J. D., Carilli, C. L., \& Troland, T. H. 2003 , ApJ, 597, 809

Morganti, R., Oosterloo, T., \& Tsvetanov, Z. 1998, AJ, 115, 915

Morganti, R., Tsvetanov, Z. I., Gallimore, J., \& Allen, M. G. 1999, A\&AS, 137, 457

Mundell, C. G., Holloway, A. J., Pedlar, A., et al. 1995, MNRAS, 275, 67

Mundell, C. G., Wilson, A. S., Ulvestad, J. S., \& Roy, A. L. 2000, ApJ, 529, 816

Murray, C., Gallimore, J. F., Baum, S. A., et al. 1999, BAAS, 31, 1544

Nagar, N. M., Falcke, H., Wilson, A. S., \& Ulvestad, J. S. 2002a, A\&A, 392, 53

Nagar, N. M., Oliva, E., Marconi, A., \& Maiolino, R. 2002b, A\&A, 391, L21

Nagar, N. M., \& Wilson, A. S. 1999, ApJ, 516, 97

Nagar, N. M., Wilson, A. S., Mulchaey, J. S., \& Gallimore, J. F. 1999, ApJS, 120, 209

Neff, S. G., \& de Bruyn, A. G. 1983, A\&A, 128, 318

Nelson, C. H., \& Whittle, M. 1995, ApJS, 99, 67

Nishiura, S., Shimada, M., Ohyama, Y., Murayama, T., \& Taniguchi, Y. 2000, AJ, 120, 1691

Norman, C., \& Miley, G. 1984, A\&A, 141, 85

Oosterloo, T. A., Morganti, R., Tzioumis, A., et al. 2000, AJ, 119, 2085

Peacock, J. A., Miller, L., \& Longair, M. S. 1986, MNRAS, 218, 265

Pearson, T. J., \& Readhead, A. C. S. 1988, ApJ, 328, 114

Peck, A. B., Henkel, C., Ulvestad, J. S., et al. 2003, ApJ, 590, 149 
Pedlar, A., Ghataure, H. S., Davies, R. D., et al. 1990, MNRAS, 246, 477

Pedlar, A., Kukula, M. J., Longley, D. P. T., et al. 1993, MNRAS, 263, 471

Pedlar, A., Unger, S. W., \& Dyson, J. E. 1985, MNRAS, 214, 463

Polatidis, A. G., Conway, J. E., \& Owsianik, I. 2002, in Proceedings of the 6th European VLBI Network Symposium, ed. E. Ros, R. W. Porcas, A. P. Lobanov, \& J. A. Zensus (Bonn: MPIfR), 139

Pringle, J. 1997, MNRAS, 292, 136

Pringle, J. E., Antonucci, R. R. J., Clarke, C. J., et al. 1999, ApJ, 526, L9

Roy, A. L., Colbert, E. J. M., Wilson, A. S., \& Ulvestad, J. S. 1998, ApJ, 504, 147

Roy, A. L., Wilson, A. S., Ulvestad, J. S., \& Colbert, J. M. 2000, in EVN Symp. 2000, Proceedings of the 5th european VLBI Network Symposium held at Chalmers University of Technology, Gothenburg, Sweden, June 29-July 1, ed. J. E. Conway, A. G. Polatidis, R. S. Booth, \& Y. M. Pihlström, published Onsala Space Observatory, 7

Rush, B., Malkan, M. A., \& Edelson, R. A. 1996, ApJ, 473, 130

Sanghera, H. S., Saikia, D. J., Luedke, E., et al. 1995, A\&A, 295, 629

Sawada-Satoh, S., Inoue, M., Shibata, K. M., et al. 2000, PASJ, 52, 421

Schmitt, H. R., \& Kinney, A. L. 2000, ApJS, 128, 479

Schmitt, H. R., Ulvestad, J. S., \& Antonucci, R. R. J. 2002, Am. Astron. Soc. Meet., 34, 715

Storchi-Bergmann, T., Fernandes, R. C., \& Schmitt, H. R. 1998, ApJ, 501, 94

Su, B. M., Muxlow, T. W. B., Pedlar, A., et al. 1996, MNRAS, 279, 1111

Taylor, D., Dyson, J. E., Axon, D. J., \& Pedlar, A. 1989, MNRAS, 240, 487

Tran, H. D. 1995, ApJ, 440, 578

Tran, H. D., Miller, J. S., \& Kay, L. E. 1992, ApJ, 397, 452

Trotter, A. S., Greenhill, L. J., Moran, J. M., et al. 1998, ApJ, 495, 740

Ulvestad, J. S., \& Ho, L. C. 2001a, ApJ, 558, 561

Ulvestad, J. S., \& Ho, L. C. 2001b, ApJ, 562, L133

Ulvestad, J. S., Neff, S. G., \& Wilson, A. S. 1987, AJ, 93, 22
Ulvestad, J. S., Roy, A. L., Colbert, E. J. M., \& Wilson, A. S. 1998, ApJ, 496, 196

Ulvestad, J. S., \& Wilson, A. S. 1983, ApJ, 264, L7

Ulvestad, J. S., \& Wilson, A. S. 1984, ApJ, 285, 439

Ulvestad, J. S., \& Wilson, A. S. 1989, ApJ, 343, 659

Ulvestad, J. S., Wong, D. S., Taylor, G. B., Mundell, C. G., \& Gallimore, J. W. 2002, Am. Astron. Soc. Meet., 201, 0

Ulvestad, J. S., Wrobel, J. M., \& Carilli, C. L. 1999a, ApJ, 516, 127

Ulvestad, J. S., Wrobel, J. M., Roy, A. L., et al. 1999b, ApJ, 517, L81

Unger, S. W., Lawrence, A., Wilson, A. S., Elvis, M., \& Wright, A. E. 1987, MNRAS, 228, 521

Unger, S. W., Pedlar, A., Axon, D. J., et al. 1988, MNRAS, 234, 745

Unger, S. W., Pedlar, A., Booler, R. V., \& Harrison, B. A. 1986 , MNRAS, 219, 387

Véron-Cetty, M.-P., \& Véron, P. 1991, A Catalogue of quasars and active nuclei ESO Scientific Report (Garching: European Southern Observatory (ESO)), 5th ed.

Véron-Cetty, M.-P., \& Véron, P. 1998, A Catalogue of quasars and active nuclei 8th ed. (Garching: European Southern Observatory (ESO)), ESO Scientific Report Series, 18

Véron-Cetty, M.-P., \& Véron, P. 2001, A\&A, 374, 92

Vermeulen, R. C., Ros, E., Kellermann, K. I., et al. 2003, A\&A, 401, 113

Vir Lal, V. 2001, Ph.D. Thesis, Indian Institute of Astrophysics, Bangalore, India

Walker, R. C., Dhawan, V., Romney, J. D., Kellermann, K. I., \& Vermeulen, R. C. 2000, ApJ, 530, 233

White, R. L., Becker, R. H., Gregg, M. D., et al. 2000, ApJS, 126, 133

Whittle, M. 1992, ApJ, 387, 121

Whittle, M., Haniff, C. A., Ward, M. J., et al. 1986, MNRAS, 222, 189

Whittle, M., Pedlar, A., Meurs, E. J. A., et al. 1988, ApJ, 326, 125

Wilson, A. S., \& Colbert, E. J. M. 1995, ApJ, 438, 62

Wilson, A. S., Penston, M. V., Fosbury, R. A. E., \& Boksenberg, A. 1976, MNRAS, 177, 673

Wilson, A. S., Roy, A. L., Ulvestad, J. S., et al. 1998, ApJ, 505, 587

Wilson, A. S., \& Tsvetanov, Z. I. 1994, AJ, 107, 1227

Wrobel, J. M. 2000, ApJ, 531, 716

Wrobel, J. M., Fassnacht, C. D., \& Ho, L. C. 2001, ApJ, 553, L23 